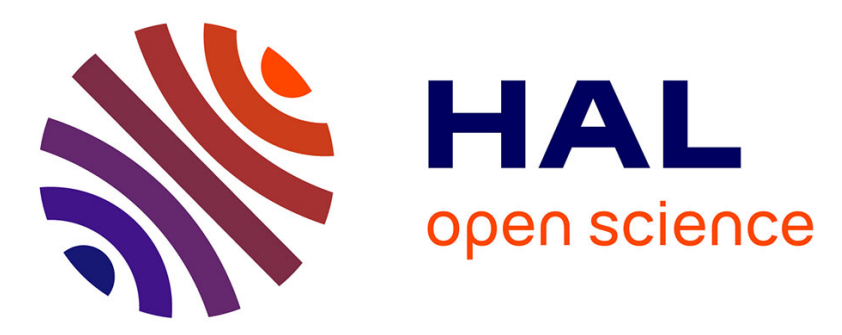

\title{
Robustness and performance analysis of subspace-based DOA estimation for rectilinear correlated sources in CES data model
}

\author{
Habti Abeida, Jean-Pierre Delmas
}

\section{- To cite this version:}

Habti Abeida, Jean-Pierre Delmas. Robustness and performance analysis of subspace-based DOA estimation for rectilinear correlated sources in CES data model. Signal Processing, 2021, 178, pp.107799:1107799:12. 10.1016/j.sigpro.2020.107799 . hal-03036485

\section{HAL Id: hal-03036485 \\ https://hal.science/hal-03036485}

Submitted on 2 Dec 2020

HAL is a multi-disciplinary open access archive for the deposit and dissemination of scientific research documents, whether they are published or not. The documents may come from teaching and research institutions in France or abroad, or from public or private research centers.
L'archive ouverte pluridisciplinaire HAL, est destinée au dépôt et à la diffusion de documents scientifiques de niveau recherche, publiés ou non, émanant des établissements d'enseignement et de recherche français ou étrangers, des laboratoires publics ou privés. 


\title{
Robustness and performance analysis of
}

\section{subspace-based DOA estimation for rectilinear correlated sources in CES data model}

\author{
Habti Abeida and Jean-Pierre Delmas
}

\begin{abstract}
This paper focuses on a theoretical performance analysis of subspace-based algorithms for the localization of spatially correlated rectilinear sources embedded in circular complex elliptically symmetric (C-CES) distributed noise model and also when the observations are non-circular CES (NC-CES) distributed with dependent scatter matrices on the direction of arrival (DOA) parameters. A perturbation analysis has been performed to derive closed-form expressions for the asymptotic covariance matrices of DOA estimates for non-circular subspacebased algorithms in two CES data models. Robustness of subspace-based algorithms is theoretical evaluated using robust covariance matrix estimators (instead of the sample covariance matrix (SCM)). We prove, for the first time, interpretable closed-form expressions of the asymptotic variance of the estimated DOA of two equi-power correlated sources, which allows us to derive a number of properties describing the DOA variance's dependence on signals parameters and non-Gaussian distribution of the noise. Different robustness properties are theoretically analyzed. In particular, we prove in the framework of NC-CES distributed observations, that Tyler's $M$-estimator enhances the performance for heavy-tailed distributions w.r.t. the SCM, with negligible loss in performance for circular Gaussian distributed observations. Finally, some Monte Carlo illustrations are given for quantifying this robustness and specifying the domain of validity of our theoretical asymptotic results.
\end{abstract}

\section{Index Terms}

Subspace-based algorithm, non-circular MUSIC algorithm, direction-of-arrival, correlated sources, rectilinear sources, strictly non-circular, complex elliptically symmetric distribution, complex generalized Gaussian distribution, non-circular M-estimators.

Paper accepted to Signal Processing

Habti Abeida is with Dept. of Electrical Engineering, University of Taif, Al-Haweiah, 21974, Saudi Arabia, email: abeida3@yahoo.fr, phone: +(996).583.367.467. Jean Pierre Delmas is with Samovar laboratory, Telecom SudParis, Institut Polytechnique de Paris, 91011 Evry Cedex, France, e-mail: jean-pierre.delmas@it-sudparis.eu, phone: +(33).1.60.76.46.32, fax: +(33).1.60.76.44.33. 


\section{INTRODUCTION}

Far and near-field narrowband source localization have received considerable attention over the last two decades (see e.g., [1], [2]). Most of the existing algorithms concentrate on the second-order statistics of the observations. Originally these algorithms were designed to process complex circular signals, but then they became interested in mobile communications systems for which the modulated signals can be complex noncircular (NC). In this case, these algorithms were based not only on the sample covariance matrix but also on the complementary (or unconjugated) sample covariance matrix. The most popular among these algorithms are the subspace-based algorithms which exploit the orthogonality between a sample subspace derived from these sample covariance matrices and a parameter-dependent subspace. However, it has been proved in [3] that the gain in performance of the subspace-based algorithms build from these two covariance matrices was significant only in the particular case of rectilinear (called also strictly non-circular or with a non-circularity rate equal to 1) signals, such as binary phase shift keying (BPSK) and de-rotated offset quadrature phase shift keying (OKPSK) modulations.

Many studies of subspace-based algorithms have focused on narrowband NC signal sources in the presence of spatially white circular complex Gaussian (C-CG) noise. In particular, NC MUSIC [4], and NC Root-MUSIC [5] algorithms have been proposed for the DOA estimation problem. A NC standard ESPRIT algorithm has also been proposed in [6] for shift-invariant arrays, where the DOA estimates are directly given instead of being found with search over the DOA space. Then a NC unitary ESPRIT algorithm that does not require a centro-symmetric array structure, but only the shift-invariance property with reduced computational complexity has been introduced in [7]. A performance analysis of different NC MUSIC-like algorithms in terms of variance and resolution has been presented in [8], [9] under the assumption of stochastic sources. The NC ESPRIT-like algorithms were also the subject of a performance analysis but under the assumption of deterministic sources. In particular, a gain calculation provided by the NC standard ESPRIT, compared to the standard ESPRIT has been developed in [10] for two uncorrelated sources with maximum phase separation. A comparison between NC standard and unitary ESPRIT algorithms has been investigated in [11], proving that these two algorithms have the same asymptotic performance when the signal-to-noise ratio (SNR) tends to infinite. A MUSIC-like and an ESPRIT-like algorithms under the co-existence of both circular and non-circular sources were presented in [12] and [13], respectively. To process temporally and spatially correlated rectilinear sources, a signal subspace fitting method has been proposed in [14]. In [15], a sparse representation technique has been introduced to estimate the DOA of NC signals. Note that the aforementioned papers relate to NC far-field signals, and some research works have been recently devoted to the localization estimation of near-field [16], [17], and mixed far-field and near-field [18], [19] uncorrelated NC signals. 
All of the localization estimation algorithms mentioned above are based on the SCM, and have been studied in C-CG noise environment and mainly for uncorrelated sources. But sometimes, this Gaussian assumption presents a poor approximation of underlying physics for which noise can be spiky and impulsive i.e., have heavier tails than the Gaussian distribution. In this context, the C-CES distributions and the subclass of the circular complex compound Gaussian (C-CCG) distributions (also referred to as spherically invariant random vector (SIRV)) (see e.g., [20]) are widely used in the engineering literature. Similarly, the assumption of uncorrelation of the rectilinear sources is not realistic in the presence of multipaths. In a non-Gaussian noise environment, these algorithms may perform poorly, resulting in unreliable DOA estimates. Robust subspace-based DOA estimation algorithms based on $M$-estimates of the covariance matrix rather than based on the SCM have been introduced (see e.g., [21]-[23]) to compensate for these poor performances. But these performances have been assessed only by Monte-Carlo experiments. It is the same for the algorithm [24] in which the SCM is replaced by a normalized SCM.

The aim of this paper is twofold. First, it is to show that all the NC subspace-based algorithms built from the SCM designed for uncorrelated rectilinear sources embedded in spatially white C-CG noise can be also applied for correlated rectilinear sources in the contexts of SCM estimate with C-CES noise and $M$-estimate with NC-CES observations. Second, it is to extend the asymptotic performance analysis of NC [resp., circular] MUSIC-like DOA estimation algorithms given in [8] for SCM estimate with Gaussian noise [resp., given in [26] for SCM and $M$-estimates with circular signals], to the contexts of SCM estimates with NC deterministic or stochastic sources embedded in C-CES noise and $M$-estimates with NC-CES observations. More precisely, closed-form expressions of the covariance of the asymptotic distribution of the estimated DOA for different data models are given. This allows us, in particular, to give for the first time an interpretable closed-form expression of the asymptotic variance of the estimated DOA of two equi-power correlated sources to assess the impact of the correlation of the sources and the non-Gaussian distribution of the noise.

This paper is organized as follows. Section II specifies the general array data model with correlated rectilinear sources and spatially white noise and describes the two statistical models with C-CES distributed noise and NC-CES distributed observations. It ends with a brief review of different NC MUSIC-like algorithms. Section III presents a theoretical asymptotic performance analysis of these algorithms under the two statistical models. Section IV gives interpretable closed-form expressions of the asymptotic variance of the estimated DOA of two equi-power correlated sources, which have never been reported in the literature including for circular sources. Then, some remarks and properties are derived from these expressions. Numerical illustrations of the performance of these algorithms with Monte-Carlo simulations are given in Section V. Finally, the paper is concluded in Section VI.

The following notations are used throughout the paper. Matrices and vectors are represented by bold upper 
case and bold lower case characters, respectively. Vectors are in column orientation, while $T, H$ and $*$ stand for transpose, conjugate transpose and conjugate, respectively. $\mathrm{E}($.$) , \operatorname{Det}(),. \operatorname{Tr}(),.(.)^{\#}$ are the expectation, determinant, trace and Moore-Penrose inverse, respectively. $\operatorname{vec}(\cdot)$ is the vectorization operator that turns a matrix into a vector by stacking the columns of the matrix one below another which is used in conjunction with the Kronecker product $\mathbf{A} \otimes \mathbf{B}$ as the block matrix whose $(i, j)$ block element is $a_{i, j} \mathbf{B}$ and with the vec-permutation matrix $\mathbf{K}$ which transforms $\operatorname{vec}(\mathbf{C})$ to $\operatorname{vec}\left(\mathbf{C}^{T}\right)$ for any square matrix $\mathbf{C}$. The matrix $\mathbf{J}$ is the exchange matrix $\left(\begin{array}{ll}\mathbf{0} & \mathbf{I} \\ \mathbf{I} & \mathbf{0}\end{array}\right)$ and $\odot$ denotes the element by element matrix product.

\section{DATA MODEL AND PROBLEM FORMULATION}

\section{A. Data model}

Consider $K$ zero-mean narrowband signals $\left(x_{t, k}\right)_{k=1, \ldots, K}$ impinging on an arbitrary array of $N$ sensors. These signals are supposed rectilinear (also called strictly second-order non-circular), i.e., described by the following model:

$$
x_{t, k}=s_{t, k} e^{i \phi_{k}} \quad \text { with } s_{t, k} \text { real-valued, }
$$

where the phases $\phi_{k}$ associated with different propagation delays are assumed fixed, but unknown during the array observation. The array output at time $t$ is modeled as

$$
\mathbf{y}_{t}=\mathbf{A}_{\theta} \boldsymbol{\Delta}_{\phi} \mathbf{s}_{t}+\mathbf{n}_{t}, \quad t=1, \ldots, T
$$

where $\mathbf{A}_{\theta} \stackrel{\text { def }}{=}\left[\mathbf{a}_{1}, \ldots, \mathbf{a}_{K}\right]$ denotes the steering matrix, where each vector $\mathbf{a}_{k}$ is parameterized in a simplified case $^{1}$ by a single real scalar parameter $\theta_{k}$ (with $\left\|\mathbf{a}_{k}\right\|$ not depending on $\theta_{k}$ ) and $\boldsymbol{\Delta}_{\phi} \stackrel{\text { def }}{=} \operatorname{Diag}\left(e^{i \phi_{1}}, \ldots, e^{i \phi_{K}}\right)$. $\mathbf{s}_{t} \stackrel{\text { def }}{=}\left(s_{t, 1}, \ldots, s_{t, K}\right)^{T}$ where $\left(s_{t, k}\right)_{k=1, \ldots, K, t=1, . . T}$ are either real-valued deterministic unknown parameters (in the so-called conditional or deterministic model), with sample covariance matrix $\mathbf{R}_{s, T}=\frac{1}{T} \sum_{t=1}^{T} \mathbf{s}_{t} \mathbf{s}_{t}^{T}$ (where $\lim _{T \rightarrow \infty} \mathbf{R}_{s, T} \stackrel{\text { def }}{=} \mathbf{R}_{s}$ exists) or zero-mean real-valued with finite fourth-order moments of arbitrary distribution and with covariance $\mathrm{E}\left(\mathbf{s}_{t} \mathbf{s}_{t}^{T}\right)=\mathbf{R}_{s}$ (in the so-called unconditional or stochastic model). Unlike previous works, we assume here that $\mathbf{R}_{s}$ is unknown non-singular. $\left(\mathbf{y}_{t}\right)_{t=1, \ldots, T}$ are independent and $\left(\mathbf{n}_{t}\right)_{t=1, \ldots, T}$ is the additive noise, which is uncorrelated with $\left(s_{t, k}\right)_{t=1, . ., T, k=1, . ., K}$ and assumed zero-mean C-CES or C-CCG distributed with finite fourth-order moments, spatially uncorrelated with $\mathrm{E}\left(\mathbf{n}_{t} \mathbf{n}_{t}^{H}\right)=\boldsymbol{\Sigma}=\sigma_{n}^{2} \mathbf{I}$. Using the stochastic representation theorem of these distributions (see e.g., [20, th.3 and def.3]), $\mathbf{n}_{t}$ is distributed as

$$
\sqrt{\mathcal{Q}_{t}} \boldsymbol{\Sigma}^{1 / 2} \mathbf{u}_{t} \text { for C-CES distributions, } \quad \sqrt{\tau_{t}} \boldsymbol{\Sigma}^{1 / 2} \mathbf{w}_{t} \text { for C-CCG distributions, }
$$

\footnotetext{
${ }^{1}$ The extension to several parameters as DOAs (azimuth, elevation), range or polarization is straightforward.
} 
where $\mathcal{Q}_{t}$ and $\tau_{t}$ are non-negative real random variables, $\mathbf{u}_{t}$ and $\mathbf{w}_{t}$ are respectively uniformly distributed on the unit complex $N$-sphere and zero-mean C-CG distributed with covariance $\mathbf{I}, \mathcal{Q}_{t}\left[\right.$ resp., $\tau_{t}$ ] and $\mathbf{u}_{t}$ [resp., $\mathbf{w}_{t}$ ] are independent and $\boldsymbol{\Sigma}$ is the scatter matrix of the distribution of $\mathbf{n}_{t}$. It is proved in [26] that the fourth-order moments of $\mathbf{n}_{t}$ are characterized by the parameter $\eta$ defined by

$$
\eta=\frac{\mathrm{E}\left(\mathcal{Q}_{t}^{2}\right)}{N(N+1)}\left[\text { resp., } \eta=\mathrm{E}\left(\tau_{t}^{2}\right)\right] \text { for C-CES [resp., C-CCG] distributions, }
$$

for which $\eta=1$ for C-CG distribution of $\mathbf{n}_{t}$. We note that $\mathrm{E}\left(\mathcal{Q}_{t}\right)=N, \mathrm{E}\left(\tau_{t}\right)=1$ and the Cauchy-Schwarz inequality implies:

$$
\eta \geq \frac{N}{N+1}[\text { resp., } \eta \geq 1] \text { for C-CES [resp., C-CCG] distributions. }
$$

To derive subspace-based algorithms exploiting the prior knowledge of rectilinear sources, the model (2) can be rewritten according to the following equivalent extended model:

$$
\widetilde{\mathbf{y}}_{t} \stackrel{\text { def }}{=}\left[\begin{array}{l}
\mathbf{y}_{t} \\
\mathbf{y}_{t}^{*}
\end{array}\right]=\widetilde{\mathbf{A}} \mathbf{s}_{t}+\widetilde{\mathbf{n}}_{t}, \quad t=1, \ldots, T,
$$

where $\widetilde{\mathbf{A}} \stackrel{\text { def }}{=}\left[\begin{array}{c}\mathbf{A}_{\theta} \boldsymbol{\Delta}_{\phi} \\ \mathbf{A}_{\theta}^{*} \boldsymbol{\Delta}_{\phi}^{*}\end{array}\right]=\left[\widetilde{\mathbf{a}}_{1}, \ldots, \widetilde{\mathbf{a}}_{K}\right]$ with $\widetilde{\mathbf{a}}_{k} \stackrel{\text { def }}{=}\left[\mathbf{a}_{k}^{T} e^{i \phi_{k}}, \mathbf{a}_{k}^{H} e^{-i \phi_{k}}\right]^{T}$ and $\widetilde{\mathbf{n}}_{t} \stackrel{\text { def }}{=}\left(\mathbf{n}_{t}^{T}, \mathbf{n}_{t}^{H}\right)^{T}$. Consequently the covariance matrix of the extended signal $\tilde{\mathbf{y}}_{t}$ is given by:

$$
\mathbf{R}_{\tilde{y}} \stackrel{\text { def }}{=} \mathrm{E}\left(\widetilde{\mathbf{y}}_{t} \widetilde{\mathbf{y}}_{t}^{H}\right)=\widetilde{\mathbf{A}} \mathbf{R}_{s} \widetilde{\mathbf{A}}^{H}+\sigma_{n}^{2} \mathbf{I} \stackrel{\text { def }}{=} \widetilde{\mathbf{S}}+\sigma_{n}^{2} \mathbf{I},
$$

assuming that the $2 N \times K$ (with $K<2 N$ ) matrix $\widetilde{\mathbf{A}}$ is of full column rank, whose subspace generated by its columns characterizes the DOAs $\left(\theta_{1}, \ldots, \theta_{K}\right)$. This condition applies to many array structures including some sparse linear arrays (see e.g., [27], [28]). Consequently, all the NC subspace-based algorithms proposed in the literature in the specific case of uncorrelated rectilinear sources (e.g., NC MUSIC-like algorithms [8] and NC ESPRIT-like algorithms [6], [7]), also apply to arbitrary noncoherent rectilinear sources. In particular the NC MUSIC-like algorithms can be considered as the following mapping derived from the extended SCM:

$$
\mathbf{R}_{\tilde{y}, T}=\frac{1}{T} \sum_{t=1}^{T} \widetilde{\mathbf{y}}_{t} \widetilde{\mathbf{y}}_{t}^{H} \longmapsto \boldsymbol{\Pi}_{\tilde{y}, T} \stackrel{\operatorname{alg}}{\longmapsto} \widehat{\boldsymbol{\theta}}_{T}=\left(\widehat{\theta}_{1, T}, \ldots, \widehat{\theta}_{K, T}\right)^{T},
$$

where $\Pi_{\tilde{y}, T}$ denotes the orthogonal projection matrix associated with the so-called noise subspace of $\mathbf{R}_{\tilde{y}, T}$ (built from the SVD of $\left.\mathbf{R}_{\tilde{y}, T}\right)$. The functional dependence $\widehat{\boldsymbol{\theta}}_{T}=\operatorname{alg}\left(\boldsymbol{\Pi}_{\tilde{y}, T}\right)$ constitutes an extension of the mapping $\boldsymbol{\Pi}_{\tilde{y}}=\mathbf{I}-\widetilde{\mathbf{A}}\left(\widetilde{\mathbf{A}} \widetilde{\mathbf{A}}^{H}\right)^{-1} \widetilde{\mathbf{A}} \stackrel{\text { alg }}{\longmapsto} \boldsymbol{\theta}$ in the neighborhood of $\boldsymbol{\Pi}_{\tilde{y}}$. Each extension alg specifies a particular subspace algorithm. 


\section{B. Robust distribution model}

To mitigate the loss of performance of subspace-based algorithms for heavy-tailed C-CES distributed noise, the extended SCM can be replaced by the ML estimate of $\mathbf{R}_{\tilde{y}}$. However, this estimate cannot be obtained for arbitrary distributed $\mathbf{s}_{t}$ and arbitrary C-CES distributed $\mathbf{n}_{t}$ in (2). To overcome this difficulty, we consider here an alternative model where the observations $\mathbf{y}_{t}$ in (2) are independent zero-mean NC-CES [25] identically distributed, with extended scatter matrix $\boldsymbol{\Gamma}_{\tilde{y}}$ whose p.d.f. is ${ }^{2}$ :

$$
p\left(\mathbf{y}_{t}\right)=\left|\boldsymbol{\Gamma}_{\tilde{y}}\right|^{-1 / 2} g\left(\frac{1}{2} \tilde{\mathbf{y}}_{t}^{H} \boldsymbol{\Gamma}_{\tilde{y}}^{-1} \tilde{\mathbf{y}}_{t}\right),
$$

where the function $g():. \mathbb{R}^{+} \mapsto \mathbb{R}^{+}$satisfies $\delta_{N, g} \stackrel{\text { def }}{=} \int_{0}^{\infty} t^{N-1} g(t) d t<\infty$. The r.v. $\mathbf{y}_{t}$ admits the following stochastic representation [29]:

$$
\mathbf{y}_{t}={ }_{d} \sqrt{\mathcal{Q}_{t}}[\mathbf{I}, \mathbf{0}] \boldsymbol{\Gamma}_{\tilde{y}}^{1 / 2} \tilde{\mathbf{u}}_{t}
$$

where $\tilde{\mathbf{u}}_{t} \stackrel{\text { def }}{=}\left(\mathbf{u}_{t}^{T}, \mathbf{u}_{t}^{H}\right)^{T}, \mathcal{Q}_{t}$ and $\mathbf{u}_{t}$ are independent, $\mathbf{u}_{t}$ is uniformly distributed on the unit complex $N$-sphere and $\mathcal{Q}_{t}$ has the p.d.f.

$$
p\left(\mathcal{Q}_{t}\right)=\delta_{N, g}^{-1} \mathcal{Q}_{t}^{N-1} g\left(\mathcal{Q}_{t}\right)
$$

Furthermore, to remove the so-called scale ambiguity, the density generator $g$ is here constrained such that $\delta_{N+1, g} / \delta_{N, g}=N$, or equivalently $\mathrm{E}\left(\mathcal{Q}_{t}\right)=N$ given the 2nd-oder moments exist [20] to ensure that the extended scatter matrix $\boldsymbol{\Gamma}_{\tilde{y}}$ is equal to the structured extended covariance matrix $\mathbf{R}_{\tilde{y}}$ in (7).

The ML estimate of $\mathbf{R}_{\tilde{y}}$ in this model is solution of the implicit equation:

$$
\boldsymbol{\Gamma}_{\tilde{y}, T}=\frac{1}{T} \sum_{t=1}^{T} \psi\left(\frac{1}{2} \tilde{\mathbf{y}}_{t}^{H} \boldsymbol{\Gamma}_{\tilde{y}, T}^{-1} \tilde{\mathbf{y}}_{t}\right) \tilde{\mathbf{y}}_{t} \tilde{\mathbf{y}}_{t}^{H},
$$

where

$$
\psi(t) \stackrel{\text { def }}{=}-\frac{1}{g(t)} \frac{d g(t)}{d t}
$$

and it is proved in [26] that the solution $\boldsymbol{\Gamma}_{\tilde{y}, T}$ of (12) converges in probability to $\mathbf{R}_{\tilde{y}}$ and can be derived from the fix point algorithm, given any positive definite Hermitian matrix $\boldsymbol{\Gamma}_{\tilde{y}, 0}$ and mild regularity conditions on $\left(\mathbf{y}_{1}, \ldots, \mathbf{y}_{T}\right)$ similarly as for the RES distribution [30].

When the density generator $g($.$) is unknown, M$-estimators have been proposed to estimate $\mathbf{R}_{\tilde{y}}$ which are also solutions of the implicit equation (12), where $\psi($.$) in (12) is replaced by a real-valued non-negative weight$ function $u($.$) which is not related to a particular NC-CES distribution. Tyler's and Huber's M$-estimators are

\footnotetext{
${ }^{2}$ This expression given in [29] is consistent with the one given in [25], because the normalizing constant is included in the function $g$.
} 
examples of such estimators (see e.g., [20, sec.V.C]). Existence and uniqueness of the solution $\boldsymbol{\Gamma}_{\tilde{y}, T}^{u}$ of (12) (where $\psi($.$) is replaced by u($.$) ) have been proved for RES distributions, provided that u($.$) satisfies a set of$ general conditions (called Maronna conditions) stated by Maronna in [31]. These conditions have been extended to C-CES distributions in [22] and [20]. Under these conditions, it has been also proved for RES distributions, that the solution of (12) can be derived by an iterative fix point algorithm [30] and converges in probability to a matrix proportional to the scatter matrix. Using an equivalence between RES and NC-CES distributions, theses properties have been extended to NC-CES distributions [29]. The sequence $\Gamma_{\tilde{y}, T}^{u}$ of solutions of (12) converges in probability to $\Gamma_{\tilde{y}}^{u}$ proportional to $\mathbf{R}_{\tilde{y}}[20,(45)]$ :

$$
\boldsymbol{\Gamma}_{\tilde{y}}^{u}=\sigma_{u} \mathbf{R}_{\tilde{y}}=\sigma_{u} \boldsymbol{\Gamma}_{\tilde{y}}
$$

where $\sigma_{u}$ depending on $u($.$) and the NC-CES distribution of \mathbf{y}_{t},[29]$ is solution of

$$
\mathrm{E}\left[u\left(\mathcal{Q}_{t} / \sigma_{u}\right) \mathcal{Q}_{t} / \sigma_{u}\right]=N
$$

Note that Tyler's $M$-estimator is also solution of (12) with weight $u(t)=\frac{N}{t}$, does not satisfy Maronna conditions [31]. It is a distribution-free estimator within the family of CES distributions. However, it has been proved for RES distributions in [32] and for C-CES distributions in [33], then extended to NC-CES distributions in [29], that after normalizing, the solution $\boldsymbol{\Gamma}_{\tilde{y}, T}^{u}$ of (12) converges in probability to $\boldsymbol{\Gamma}_{\tilde{y}}^{u}=\mathbf{R}_{\tilde{y}}$, i.e., satisfies (14) with $\sigma_{u}=1$.

With this new model, all the subspace-based (MUSIC or ESPRIT) algorithms proposed in the literature in the specific case of uncorrelated rectilinear or deterministic rectilinear sources, also apply to arbitrary noncoherent rectilinear sources by replacing in (8) the SCM matrix $\mathbf{R}_{\tilde{y}, T}$ by $\boldsymbol{\Gamma}_{\tilde{y}, T}^{u}$.

\section{Subspace-based estimation}

We specify some examples of such NC MUSIC-like algorithms built from $\Pi_{\tilde{y}, T}$ which is structured [8] as:

$$
\boldsymbol{\Pi}_{\tilde{y}, T}=\left(\begin{array}{ll}
\boldsymbol{\Pi}_{1, T} & \boldsymbol{\Pi}_{2, T} \\
\boldsymbol{\Pi}_{2, T}^{*} & \boldsymbol{\Pi}_{1, T}^{*}
\end{array}\right),
$$

where $\boldsymbol{\Pi}_{1, T}$ and $\boldsymbol{\Pi}_{2, T}$ are Hermitian and complex symmetric matrices, respectively. The following three NC MUSIC-like algorithms introduced firstly in [4], [8] and [5] for uncorrelated sources, respectively, can fully apply without any changes to the models presented above. Specifically, the estimated DOA $\left(\widehat{\theta}_{k, T}\right)_{k=1, \ldots, K}$ given 
by the first two algorithms are obtained as the locations the $K$ smallest minima of localization functions:

$$
\begin{aligned}
\widehat{\theta}_{k, T}^{\operatorname{alg}} & =\arg \min _{\theta} \mathbf{a}^{H}(\theta) \mathbf{\Pi}_{1, T} \mathbf{a}(\theta)-\left|\mathbf{a}^{T}(\theta) \boldsymbol{\Pi}_{2, T}^{*} \mathbf{a}(\theta)\right|, \\
\widehat{\theta}_{k, T}^{\mathrm{alg}} & =\arg \min _{\theta}\left(\mathbf{a}^{H}(\theta) \boldsymbol{\Pi}_{1, T} \mathbf{a}(\theta)\right)^{2}-\left(\mathbf{a}^{T}(\theta) \mathbf{\Pi}_{2, T}^{*} \mathbf{a}(\theta)\right)\left(\mathbf{a}^{H}(\theta) \mathbf{\Pi}_{2, T} \mathbf{a}^{*}(\theta)\right),
\end{aligned}
$$

where $\mathbf{a}(\theta)$ denotes the parametrized steering vector. For the third algorithm introduced in [5], the estimated DOAs are given by the arguments of the roots of a polynomial:

$$
\begin{aligned}
& \widehat{\theta}_{k, T}^{\operatorname{alg} g_{3}}=\arg \left(z_{k}\right) \quad \text { with } z_{k} K \operatorname{roots}_{|z|<1} \text { of } \\
& \quad\left(\mathbf{a}^{T}\left(z^{-1}\right) \boldsymbol{\Pi}_{1, T} \mathbf{a}(z)\right)^{2}-\left(\mathbf{a}^{T}(z) \boldsymbol{\Pi}_{2, T}^{*} \mathbf{a}(z)\right)\left(\mathbf{a}^{T}\left(z^{-1}\right) \boldsymbol{\Pi}_{2, T} \mathbf{a}\left(z^{-1}\right)\right) \text { closest to the unit circle, }
\end{aligned}
$$

with $\mathbf{a}(z) \stackrel{\text { def }}{=}\left(1, z, \ldots, z^{N-1}\right)^{T}$, in the particular case of uniform linear arrays of generic steering vectors $\mathbf{a}(\theta)=\left(1, e^{i \theta}, \ldots, e^{i(N-1) \theta}\right)^{T}$ with $\theta=\pi \sin \omega$, where $\omega$ is the DOA relative to the normal of array broadside. Note that this NC root MUSIC algorithm also applies to nonuniform linear arrays whose sensors are located on a uniform grid with missing sensors such that there is no ambiguity, for which $\mathbf{a}(z) \stackrel{\text { def }}{=}\left(1, z^{c_{1}}, \ldots, z^{c_{N-1}}\right)^{T}$ where $c_{1}, \ldots, c_{N-1}$ are integers, associated with $\mathbf{a}(\theta)=\left(1, e^{i c_{1} \theta}, \ldots, e^{i c_{N-1} \theta}\right)^{T}$. Also note that the NC root MUSIC algorithm would apply for example to nested and coprime arrays for the case where the number of rectilinear sources is strictly less than $2 N$ whether the sources are correlated or not.

\section{STATISTICAL PERFORMANCE OF NON-CIRCULAR MUSIC-LIKE ALGORITHMS}

\section{A. Asymptotic distribution of DOA estimates for C-CES distributed noise}

This subsection is devoted to the theoretical studies of the asymptotic performance of NC MUSIC-like DOA estimation algorithms presented in Subsection II-C for C-CES distributed noise model given in (3). Following a similar approach to those presented in [8] and [26], the following result is proved in Appendix:

Result 1: In the stochastic and deterministic models presented in Subsection II-A, the sequences $\sqrt{T}\left(\widehat{\boldsymbol{\theta}}_{T}-\boldsymbol{\theta}\right)$, where $\widehat{\boldsymbol{\theta}}_{T}$ are the DOA estimates given by the three NC MUSIC-like algorithms (17), (18) and (19), converge in distribution to the same zero-mean Gaussian distribution with covariance matrix:

$$
\mathbf{R}_{\mathrm{NG}}(\theta)=\underbrace{\widetilde{\mathbf{H}} \odot\left(\widetilde{\mathbf{A}}^{H} \widetilde{\mathbf{U}} \widetilde{\mathbf{A}}\right)}_{\mathbf{R}_{\mathrm{G}}(\theta)}+(\eta-1)\left[\widetilde{\mathbf{H}} \odot\left(\widetilde{\mathbf{A}}^{H} \widetilde{\mathbf{U}}^{\prime} \widetilde{\mathbf{A}}\right)\right],
$$

where $\mathbf{R}_{\mathrm{G}}(\theta)$ is the asymptotic covariance matrix of DOA estimates for C-CG distributed noise and $\eta$ is a fourth-order noise parameter defined by (4) which takes the unit value for C-CG distributed noise. $\widetilde{\mathbf{U}} \stackrel{\text { def }}{=}$ $\sigma_{n}^{2} \widetilde{\mathbf{S}}^{\#}+\sigma_{n}^{4}\left(\widetilde{\mathbf{S}}^{\#}\right)^{2}, \widetilde{\mathbf{U}}^{\prime} \stackrel{\text { def }}{=} \sigma_{n}^{4}\left(\widetilde{\mathbf{S}}^{\#}\right)^{2}$ and $\widetilde{\mathbf{H}}$ is a purely geometric and phase matrix such that:

$$
[\widetilde{\mathbf{H}}]_{k, l}=\frac{2}{\tilde{\gamma}_{k} \tilde{\gamma}_{l}}\left(\tilde{\alpha}_{\phi, \phi}^{(k, k)} \tilde{\alpha}_{\theta, \theta}^{(k, l)} \tilde{\alpha}_{\phi, \phi}^{(l, l)}-\tilde{\alpha}_{\phi, \phi}^{(k, k)} \tilde{\alpha}_{\theta, \phi}^{(k, l)} \tilde{\alpha}_{\theta, \phi}^{(l i l)}-\tilde{\alpha}_{\theta, \phi}^{(k, k)} \tilde{\alpha}_{\phi, \theta}^{(k, l)} \tilde{\alpha}_{\phi, \phi}^{(l, l)}+\tilde{\alpha}_{\theta, \phi}^{(k, k)} \tilde{\alpha}_{\phi, \phi}^{(k, l)} \tilde{\alpha}_{\theta, \phi}^{(l, l)}\right),
$$


with $\tilde{\alpha}_{i, j}^{(k, l)} \stackrel{\text { def }}{=} 2 \tilde{\mathbf{a}}_{i, k}^{\prime H} \boldsymbol{\Pi}_{\tilde{y}} \tilde{\mathbf{a}}_{j, l}^{\prime}, i, j=\theta, \phi$ where $\tilde{\mathbf{a}}_{\theta, k}^{\prime} \stackrel{\text { def }}{=} \frac{d \tilde{\mathbf{a}}_{k}}{d \theta_{k}}, \tilde{\mathbf{a}}_{\phi, k}^{\prime} \stackrel{\text { def }}{=} \frac{d \tilde{\mathbf{a}}_{k}}{d \phi_{k}}$ and $\tilde{\gamma}_{k} \stackrel{\text { def }}{=} \tilde{\alpha}_{\theta, \theta}^{(k, k)} \tilde{\alpha}_{\phi, \phi}^{(k, k)}-\left(\tilde{\alpha}_{\theta, \phi}^{(k, k)}\right)^{2}$. It follows from (21) that the diagonals of $\mathbf{R}_{\mathrm{NG}}(\theta)$ give the asymptotic variances:

$$
\left[\mathbf{R}_{\mathrm{NG}}(\theta)\right]_{k, k}=\underbrace{\frac{2 \tilde{\alpha}_{\phi, \phi}^{(k, k)}}{\tilde{\gamma}_{k}} \tilde{\mathbf{a}}_{k}^{H} \widetilde{\mathbf{U}} \tilde{\mathbf{a}}_{k}}_{\left.\mathbf{R}_{\mathrm{G}}(\theta)\right]_{k, k}}+(\eta-1) \frac{2 \tilde{\alpha}_{\phi, \phi}^{(k, k)}}{\tilde{\gamma}_{k}} \tilde{\mathbf{a}}_{k}^{H} \widetilde{\mathbf{U}}^{\prime} \tilde{\mathbf{a}}_{k}, \quad k=1, \ldots, K .
$$

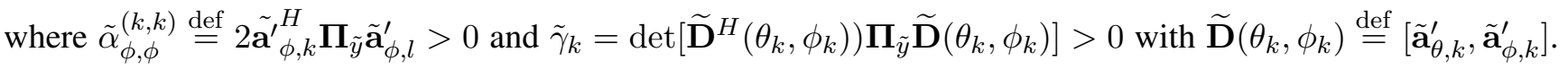

According to (21), the subspace-based algorithms are robust to the distribution of the sources, as the performance depends only on their second-order statistics, whereas the distribution of the noise can impact the performance because the non-Gaussian additive term in (23) is positive for all C-CCG noise distributions from (5). This includes in particular the circular complex Student $t$ and generalized Gaussian distributions [20] for which $\eta-1$ is very large for heavy-tailed distributions. Furthermore this additive term, which is inversely proportional to the square of the SNR, affects mainly the performance given in the Gaussian scenario at low SNR values, as it is illustrated in Section V.

For a single rectilinear source, $\widetilde{\mathbf{S}} \#=\frac{1}{\sigma_{1}^{2}} \frac{\widetilde{a}_{1} \widetilde{\mathbf{a}}_{1}^{H}}{4\left\|\mathbf{a}_{1}\right\|^{2}}$ and $\widetilde{\mathbf{U}}$ and $\widetilde{\mathbf{U}}^{\prime}$ straightforwardly follow and (23) reduces to the interpretable expression:

$$
R_{\mathrm{NG}}\left(\theta_{1}\right)=\underbrace{\frac{1}{\alpha_{1,1}}\left[\frac{1}{r_{1}}+\frac{1}{2\left\|\mathbf{a}_{1}\right\|^{2}} \frac{1}{r_{1}^{2}}\right]}_{R_{\mathrm{G}}\left(\theta_{1}\right)}+(\eta-1) \frac{1}{\alpha_{1,1}} \frac{1}{2\left\|\mathbf{a}_{1}\right\|^{2}} \frac{1}{r_{1}^{2}},
$$

where $R_{\mathrm{G}}\left(\theta_{1}\right)$ is the asymptotic variance of a single rectilinear source derived in [8] for C-CG distributed noise, where $r_{1}=\frac{\sigma_{1}^{2}}{\sigma_{n}^{2}}$ (with $\sigma_{1}^{2}$ is the power of the source) is the SNR and $\alpha_{1,1}$ is the purely geometric factor $2 \mathbf{a}_{1}^{\prime}{ }^{H} \boldsymbol{\Pi}_{y} \mathbf{a}_{1}^{\prime}$ with $\mathbf{a}_{1}^{\prime} \stackrel{\text { def }}{=} \frac{d \mathbf{a}_{1}}{d \theta_{1}}$ and $\boldsymbol{\Pi}_{y}$ is the noise subspace associated with $\mathbf{y}_{t}$.

It is important to quantify the performance gain provided by extended subspace-based algorithms that exploit noncircularity compared to conventional algorithms. This is why we consider in the following the conventional MUSIC algorithm based on $\mathbf{y}_{t}$ only for which from (2):

$$
\mathbf{R}_{y} \stackrel{\text { def }}{=} \mathrm{E}\left(\mathbf{y}_{t} \mathbf{y}_{t}^{H}\right)=\mathbf{A}_{\theta}\left(\boldsymbol{\Delta}_{\phi} \mathbf{R}_{s} \boldsymbol{\Delta}_{\phi}^{H}\right) \mathbf{A}_{\theta}^{H}+\sigma_{n}^{2} \mathbf{I} \stackrel{\text { def }}{=} \mathbf{A}_{\theta} \mathbf{R}_{x} \mathbf{A}_{\theta}^{H}+\sigma_{n}^{2} \mathbf{I} \stackrel{\text { def }}{=} \mathbf{S}+\sigma_{n}^{2} \mathbf{I} .
$$

It is worth noting that this conventional MUSIC algorithm, does not use the particular structure of the positive definite Hermitian matrix $\mathbf{R}_{x}$, so its asymptotic performance are those of the MUSIC algorithm applied to circular complex correlated sources, for which the following result has been proved in [26]:

Result 2: In the stochastic and deterministic models presented in Subsection II-A, the sequence $\sqrt{T}\left(\widehat{\boldsymbol{\theta}}_{T}-\boldsymbol{\theta}\right)$, where $\widehat{\boldsymbol{\theta}}_{T}$ are the DOA estimates given by the conventional MUSIC algorithm applied to correlated rectilinear or circular complex sources converges in distribution to the zero-mean Gaussian distribution with the same 
covariance matrix:

$$
\mathbf{R}_{\mathrm{NG}}(\theta)=\underbrace{\operatorname{Re}\left[\mathbf{H} \odot\left(\mathbf{A}_{\theta}^{H} \mathbf{U} \mathbf{A}_{\theta}\right)\right]}_{\mathbf{R}_{\mathrm{G}}(\theta)}+(\eta-1) \operatorname{Re}\left[\mathbf{H} \odot\left(\mathbf{A}_{\theta}^{H} \mathbf{U}^{\prime} \mathbf{A}_{\theta}\right)\right]
$$

with $\mathbf{R}_{\mathrm{G}}(\theta)$ is the asymptotic covariance matrix of DOA estimate for C-CG distributed noise, where $\mathbf{U} \stackrel{\text { def }}{=}$ $\sigma_{n}^{2} \mathbf{S}^{\#}+\sigma_{n}^{4}\left(\mathbf{S}^{\#}\right)^{2}, \mathbf{U}^{\prime} \stackrel{\text { def }}{=} \sigma_{n}^{4}\left(\mathbf{S}^{\#}\right)^{2}$ and $\mathbf{H}$ is a purely geometric matrix such that $[\mathbf{H}]_{k, l}=\frac{\alpha_{k, l}^{*}}{\alpha_{k, k} \alpha_{l, l}}$ with $\alpha_{k, l} \stackrel{\text { def }}{=} 2 \mathbf{a}_{k}^{\prime H} \boldsymbol{\Pi}_{y} \mathbf{a}_{l}^{\prime}$ and $\mathbf{a}_{k}^{\prime} \stackrel{\text { def }}{=} \frac{d \mathbf{a}_{k}}{d \theta_{k}}$. It follows further from (26) the asymptotic variances:

$$
\left[\mathbf{R}_{\mathrm{NG}}(\theta)\right]_{k, k}=\underbrace{\frac{1}{\alpha_{k, k}} \mathbf{a}_{k}^{H} \mathbf{U} \mathbf{a}_{k}}_{\left.\mathbf{R}_{\mathrm{G}}(\theta)\right]_{k, k}}+(\eta-1) \frac{1}{\alpha_{k, k}} \mathbf{a}_{k}^{H} \mathbf{U}^{\prime} \mathbf{a}_{k}, \quad k=1, \ldots, K .
$$

Furthermore, for a single source, (27) reduces to

$$
R_{\mathrm{NG}}\left(\theta_{1}\right)=\underbrace{\frac{1}{\alpha_{1,1}}\left[\frac{1}{r_{1}}+\frac{1}{\left\|\mathbf{a}_{1}\right\|^{2}} \frac{1}{r_{1}^{2}}\right]}_{R_{\mathrm{G}}\left(\theta_{1}\right)}+(\eta-1) \frac{1}{\alpha_{1,1}} \frac{1}{\left\|\mathbf{a}_{1}\right\|^{2}} \frac{1}{r_{1}^{2}},
$$

\section{B. Asymptotic distribution of DOA estimates for NC-CES distributed observations}

This subsection investigates the asymptotic performance of NC MUSIC-like DOA estimation algorithms presented in Subsection II-C for NC-CES distributed observations model presented in Subsection II-B. Following a similar approach to those presented in [8] and [26], the following result is proved in Appendix:

Result 3: For NC-CES distributed observations, the sequences $\sqrt{T}\left(\widehat{\boldsymbol{\theta}}_{T}-\boldsymbol{\theta}\right)$, where $\widehat{\boldsymbol{\theta}}_{T}$ are the DOA estimates given by the three NC MUSIC-like algorithms (17), (18) and (19) built from the $M$-estimate $\boldsymbol{\Gamma}_{\tilde{y}, T}$, converge in distribution to the same zero-mean Gaussian distribution with covariance matrix:

$$
\mathbf{R}_{\mathrm{NC}-\mathrm{CES}}(\theta)=\frac{\vartheta_{1}}{\sigma_{u}^{2}}\left[\widetilde{\mathbf{H}} \odot\left(\widetilde{\mathbf{A}}^{H} \widetilde{\mathbf{U}} \widetilde{\mathbf{A}}\right)\right]
$$

where $\sigma_{u}$ is solution of (15) and $\vartheta_{1}$ is given by

$$
\vartheta_{1}=\frac{\mathrm{E}\left[u^{2}\left(\mathcal{Q}_{t} / \sigma_{u}\right) \mathcal{Q}_{t}^{2}\right]}{N(N+1)\left(1+[N(N+1)]^{-1} c_{u}\right)^{2}} \text { with } c_{u} \stackrel{\text { def }}{=} \mathrm{E}\left[u^{\prime}\left(\mathcal{Q}_{t} / \sigma_{u}\right) \mathcal{Q}_{t}^{2} / \sigma_{u}^{2}\right]
$$

for the $M$-estimates satisfying Maronna conditions [31], which reduces, respectively, for ML $M$-estimates and extended SCM estimate to

$$
\vartheta_{1, \mathrm{ML}}=\frac{N(N+1)}{\mathrm{E}\left[\psi^{2}\left(\mathcal{Q}_{t}\right) \mathcal{Q}_{t}^{2}\right]}
$$

and

$$
\vartheta_{1, \mathrm{SCM}}=\eta,
$$


and to [33]

$$
\vartheta_{1, \text { Tyler }}=\frac{N+1}{N},
$$

for Tyler's $M$-estimate associated with $u(t)=\frac{N}{t}$.

Furthermore, specializing the $M$-estimates in Result 3 and its dependent parameters $\vartheta_{1}$, the following result is proved in Appendix:

Result 4: The NC-CES ML $M$-estimator dependent asymptotic covariance parameter $\vartheta_{1, \mathrm{ML}}$ (31) is upper bounded by the ones associated with Tyler's $M$-estimator (33) and with the extended SCM estimator (32) as

$$
\begin{aligned}
& \vartheta_{1, \mathrm{ML}} \leq \vartheta_{1, \mathrm{SCM}}, \\
& \vartheta_{1, \mathrm{ML}} \leq \vartheta_{1, \mathrm{Tyler}},
\end{aligned}
$$

and because $\sigma_{u}=1$ for these three $M$-estimators, (29) gives

$$
\mathbf{R}_{\mathrm{NC}-\mathrm{CES}}^{\mathrm{ML}}(\theta) \leq \mathbf{R}_{\mathrm{NC}-\mathrm{CES}}^{\mathrm{SCM}}(\theta) \text { and } \mathbf{R}_{\mathrm{NC}-\mathrm{CES}}^{\mathrm{ML}}(\theta) \leq \mathbf{R}_{\mathrm{NC}-\mathrm{CES}}^{\mathrm{Tyler}}(\theta)
$$

For example, for the NC complex Student $t$-distribution of $\nu$ degree of freedom $(0<\nu<\infty)$ which has finite second and fourth-order moments, respectively, for $\nu>2$ and $\nu>4$ (see e.g., [20, sec. IVA]), $\vartheta_{1, M L}=$ $\frac{N+\nu / 2+1}{N+\nu / 2}$ and $\vartheta_{1, \mathrm{SCM}}=\eta=\frac{\nu-2}{\nu-4}[26]$, and thus $\vartheta_{1, \mathrm{ML}} / \vartheta_{1, \mathrm{SCM}}=1-\frac{2(N+2)}{(N+\nu / 2)(\nu-2)}<1$ and $\vartheta_{1, \mathrm{ML}} / \vartheta_{1, \text { Tyler }}=$ $1-\frac{\nu / 2}{(N+\nu / 2)(N+1)}<1$. We see that $\vartheta_{1, \mathrm{ML}} / \vartheta_{1, \mathrm{SCM}} \approx 1$ for $\nu \rightarrow \infty$ (i.e., the observations tend to be $\mathrm{NC}$ Gaussian distributed) but $\vartheta_{1, \mathrm{ML}} / \vartheta_{1, \mathrm{SCM}} \ll 1$ when $\nu$ approaches 4 . This confirms that extended SCM has poor performance for heavy-tailed distributions. In contrast, for Tyler's $M$-estimator of the extended covariance matrix, $\vartheta_{1, \mathrm{ML}} / \vartheta_{1, \text { Tyler }} \approx 1$ for $N \gg 1$ and arbitrary $\nu$. Thus the NC subspace-based algorithms derived from Tyler's $M$-estimator are robust to heavy-tailed distributions. For example, for $N=5, \vartheta_{1, \mathrm{ML}} / \vartheta_{1, \mathrm{SCM}}=0.058$ and $\vartheta_{1, \mathrm{ML}} / \vartheta_{1, \mathrm{Tyler}}=0.952$ for the NC complex Student $t$-distribution with $\nu=4.1$, whereas $\vartheta_{1, \mathrm{ML}} / \vartheta_{1, \mathrm{SCM}}=1$ and $\vartheta_{1, \mathrm{ML}} / \vartheta_{1, \text { Tyler }}=0.833$ for the NC Gaussian distribution.

Note that the asymptotic performance of the NC subspace-based DOA estimation algorithms (17), (18) and (19) built from the ML $M$-estimator and the extended SCM estimator are NC-CES dependent through the parameters $\vartheta_{1}$ and $\eta$, respectively. This is in contrast to Tyler's distribution-free $M$-estimator and to an arbitrary $M$-estimator satisfying Maronna's conditions [31] for which the performances depend both on the weighting function $u(t)$ and the parameter-dependent NC-CES distribution $\vartheta_{1} / \sigma_{u}^{2}$.

\section{ASYMPTOTIC VARIANCE OF DOA ESTIMATES FOR TWO EQUI-POWERED CORRELATED SOURCES}

To derive interpretable expressions from (23), (27) and (29) for several sources, we consider in this section the particular case of two equi-power correlated sources, for which interpretable closed-form expressions for 
the asymptotic covariance matrices of DOA estimates are given for both C-CES distributed noise model and NC-CES distributed observations model.

\section{A. C-CES distributed noise model}

For the C-CES distributed noise model of Subsection II-A, the following result is proved in Appendix:

Result 5: For two equi-power correlated rectilinear sources of correlation $\rho \in(-1,1)$ of power $\sigma_{s}^{2}$ for which $\mathbf{R}_{s}=\sigma_{s}^{2}\left(\begin{array}{cc}1 & \rho \\ \rho & 1\end{array}\right)$ and SNR $r=\frac{\sigma_{s}^{2}}{\sigma_{n}^{2}}$, (23) reduces to the following interpretable expression:

$$
\left[\mathbf{R}_{\mathrm{NG}}(\theta)\right]_{k, k}=\left[\mathbf{R}_{\mathrm{G}}(\theta)\right]_{k, k}+(\eta-1) \frac{2 \tilde{\alpha}_{\phi, \phi}^{(k, k)}}{\tilde{\gamma}_{k}}\left[\left(\frac{1+\rho^{2}+2 \tilde{\beta} \rho}{\left(1-\tilde{\beta}^{2}\right)\left(1-\rho^{2}\right)^{2}}\right) \frac{1}{2\left\|\mathbf{a}_{k}\right\|^{2}} \frac{1}{r^{2}}\right], \quad k=1,2,
$$

where

$$
\left[\mathbf{R}_{\mathrm{G}}(\theta)\right]_{k, k}=\frac{2 \tilde{\alpha}_{\phi, \phi}^{(k, k)}}{\tilde{\gamma}_{k}}\left[\left(\frac{1}{1-\rho^{2}}\right) \frac{1}{r}+\left(\frac{1+\rho^{2}+2 \tilde{\beta} \rho}{\left(1-\tilde{\beta}^{2}\right)\left(1-\rho^{2}\right)^{2}}\right) \frac{1}{2\left\|\mathbf{a}_{k}\right\|^{2}} \frac{1}{r^{2}}\right], \quad k=1,2,
$$

is the asymptotic covariance matrix of DOA estimate of two equi-power correlated rectilinear sources for C-CG distributed noise, where $\tilde{\beta} \stackrel{\text { def }}{=} \frac{\tilde{\mathbf{a}}_{1}^{H} \tilde{\mathbf{a}}_{2}}{\left\|\tilde{\mathbf{a}}_{1}\right\|\left\|\tilde{\mathbf{a}}_{2}\right\|} \in(-1,1)$ is a geometric and phase factor.

To the best of our knowledge, it is interesting to note that for correlated circular complex sources, despite many experimental studies (see e.g., [34]-[36]) showing the degradation of performance of the conventional MUSIC algorithm with the correlation of the sources, no interpretable closed-form expressions of the asymptotic variances has been given up to now. This is due to the difficulty to obtain a simple interpretable closed-form expression of the Moore Penrose inverse of $\mathbf{S} \stackrel{\text { def }}{=} \mathbf{A}_{\theta} \mathbf{R}_{x} \mathbf{A}_{\theta}^{H}$ in (27). But similarly to Result 5 deduced from Result 1, the following result concerning the conventional MUSIC algorithm is proved in Appendix:

Result 6: For two equi-power correlated sources of power $\sigma_{s}^{2}$ and $\operatorname{SNR} r$, rectilinear of correlation $\rho \in(-1,1)$ for which $\mathbf{R}_{x}=\sigma_{s}^{2}\left(\begin{array}{cc}1 & \rho^{\prime} \\ \rho^{\prime *} & 1\end{array}\right)$ with $\rho^{\prime} \stackrel{\text { def }}{=} \rho e^{i\left(\phi_{1}-\phi_{2}\right)}$ in (25), or circular of correlation $\rho^{\prime} \in \mathbb{C}$ (with $\left|\rho^{\prime}\right|<1$ ) for which also $\mathbf{R}_{x}=\sigma_{s}^{2}\left(\begin{array}{cc}1 & \rho^{\prime} \\ \rho^{\prime *} & 1\end{array}\right)$, (27) reduces to the following interpretable expression:

$$
\left[\mathbf{R}_{\mathrm{NG}}(\theta)\right]_{k, k}=\left[\mathbf{R}_{\mathrm{G}}(\theta)\right]_{k, k}+(\eta-1) \frac{1}{\alpha_{k, k}}\left[\left(\frac{1+\left|\rho^{\prime}\right|^{2}+2 \operatorname{Re}\left(\beta^{*} \rho^{\prime}\right)}{\left(1-|\beta|^{2}\right)\left(1-\left|\rho^{\prime}\right|^{2}\right)^{2}}\right) \frac{1}{\left\|\mathbf{a}_{k}\right\|^{2}} \frac{1}{r^{2}}\right], \quad k=1,2,
$$

where

$$
\left[\mathbf{R}_{\mathrm{G}}(\theta)\right]_{k, k}=\frac{1}{\alpha_{k, k}}\left[\left(\frac{1}{1-\left|\rho^{\prime}\right|^{2}}\right) \frac{1}{r}+\left(\frac{1+\left|\rho^{\prime}\right|^{2}+2 \operatorname{Re}\left(\beta^{*} \rho^{\prime}\right)}{\left(1-|\beta|^{2}\right)\left(1-\left|\rho^{\prime}\right|^{2}\right)^{2}}\right) \frac{1}{\left\|\mathbf{a}_{k}\right\|^{2}} \frac{1}{r^{2}}\right], \quad k=1,2
$$

is the asymptotic covariance matrix of DOA estimates of two equi-power correlated rectilinear or circular 
complex sources for C-CG distributed noise, where $\beta \stackrel{\text { def }}{=} \frac{\mathbf{a}_{1}^{H} \mathbf{a}_{2}}{\left\|\mathbf{a}_{1}\right\|\left\|\mathbf{a}_{2}\right\|} \in \mathbb{C}$ is a purely geometric factor ${ }^{3}$

\section{B. NC-CES distributed observations model}

For NC-CES distributed observations model, we have $\mathbf{R}_{\mathrm{NC}-\mathrm{CES}}(\theta)=\frac{\vartheta_{1}}{\sigma_{u}^{2}} \mathbf{R}_{\mathrm{G}}(\theta)$ from (21) and (29), and therefore, the following result is deduced from (38).

Result 7: For NC-CES observations with two equi-power correlated rectilinear sources of correlation $\rho$ of SNR $r,(29)$ reduces to the following interpretable expression:

$$
\left[\mathbf{R}_{\mathrm{NC}-\mathrm{CES}}(\theta)\right]_{k, k}=\frac{\vartheta_{1}}{\sigma_{u}^{2}} \frac{2 \tilde{\alpha}_{\phi, \phi}^{(k, k)}}{\tilde{\gamma}_{k}}\left[\left(\frac{1}{1-\rho^{2}}\right) \frac{1}{r}+\left(\frac{1+\rho^{2}+2 \tilde{\beta} \rho}{\left(1-\tilde{\beta}^{2}\right)\left(1-\rho^{2}\right)^{2}}\right) \frac{1}{2\left\|\mathbf{a}_{k}\right\|^{2}} \frac{1}{r^{2}}\right], \quad k=1,2 .
$$

\section{General comments}

This section presents some properties of the asymptotic variances on DOA estimation in (37)-(41). It explains how asymptotic variances change as a function of different arrays, signal sources and noise parameters.

Property 1: Naturally, all the performance degrades dramatically for strongly correlated sources (i.e., $|\rho| \approx 1$ ), because in this case, the signal subspace is close to being one-dimensional for coherent sources. Also, from (39)-(40), it follows that the performance of the conventional MUSIC algorithm strongly degrades for close steering vectors $\mathbf{a}_{1}$ and $\mathbf{a}_{2}$ (i.e., $|\beta| \approx 1$ for closely-spaced sources). In contrast, for the NC subspace-based algorithms (17), (18) and (19), the performance does not necessarily collapse because

$$
\tilde{\beta}=|\beta| \cos \left(\phi_{2}-\phi_{1}+\angle \beta\right)
$$

in (37)-(38) and (41) which is equal to 1 if both $|\beta|=1$ and $\phi_{2}-\phi_{1}+\angle \beta=2 k \pi, k \in \mathbb{Z}$. Phase differences can then compensate for the closeness of the source's DOAs because increasing the degree of freedom generally improves the source resolution.

Property 2: For orthogonal steering vectors or phases in quadrature, we have $\tilde{\beta}=0$ in (37)-(38) using (42), and similarly, orthogonal steering vectors yields $\beta=0$ in (39)-(40). Thus it follows that, in both cases, the asymptotic variances on DOA estimation associated with NC subspace-based algorithms ((17), (18) and (19)) and conventional MUSIC algorithm monotonously increase with $|\rho|$ from $\rho=0$ (uncorrelated sources) to $|\rho| \approx 1$ (strongly correlated sources). But when the extended steering vectors and steering vectors are not orthogonal, the previous asymptotic variances not necessarily increase monotonously with with $|\rho|$. A figure illustrating this situation is given in Section V.

\footnotetext{
${ }^{3}$ Note that for centro-symmetric arrays (e.g., uniform linear arrays, uniform circular arrays, cross-based centro-symmetric arrays, square-based centro-symmetric array [38]), for which the array centroid is chosen as the reference of the phases, $\beta$ is real-valued and thus $\operatorname{Re}\left(\beta^{*} \rho^{\prime}\right)$ in (39) and (40) reduces to $\beta \operatorname{Re}\left(\rho^{\prime}\right)$.
} 
Regarding the dependence of algorithms performance on array SNR (i.e., $\left\|\mathbf{a}_{k}\right\|^{2} r$ ), we observe that for large array SNR, the term $\frac{1}{1-|\rho|^{2}}$ is dominant in all dependent asymptotic variances expressions (37)-(41) and thus the asymptotic variances of DOA estimation monotonously increase with $|\rho|$ for arbitrary $\tilde{\beta}$ or $\beta$. In contrast, for weak array SNR $\left(\left\|\mathbf{a}_{k}\right\|^{2} r \ll 1\right)$, the terms $\left(\frac{1+\rho^{2}+2 \tilde{\beta} \rho}{\left(1-\tilde{\beta}^{2}\right)\left(1-\rho^{2}\right)^{2}}\right) \frac{1}{2\left\|\mathbf{a}_{k}\right\|^{2} r}$ in (37)-(38), (41) and $\left(\frac{1+|\rho|^{2}+2 \operatorname{Re}\left(\beta^{*} \rho\right)}{\left(1-|\beta|^{2}\right)\left(1-|\rho|^{2}\right)^{2}}\right) \frac{1}{\left\|\mathbf{a}_{k}\right\|^{2} r}$ in (39)-(40) are dominant in (37)-(41) w.r.t. the term $\frac{1}{1-|\rho|^{2}}$ and thus the purely geometric parameter $\beta$ and statistical $\rho$ are coupled in the expressions of the asymptotic variances which do not necessarily increase with both $\tilde{\beta}$ or $\beta$ and $|\rho|$.

Property 3: For both orthogonal extended steering vectors [resp., orthogonal steering vectors] and uncorrelated sources, the asymptotic variances of DOA estimation (37)-(38) and (41) [resp., (39)-(40)] are equal to the asymptotic variance (24) [resp., (28)] given for a single source, up to a multiplicative geometric and phase-dependent factor [resp., purely geometric factor]. This factor is proved to be strictly larger than 1 for the conventional MUSIC algorithm because $\alpha_{1,1}^{2}$ orthogonal sources $=\alpha_{1,1}^{1}$ source $-2\left|\mathbf{a}_{2}^{H} \mathbf{a}_{1}^{\prime}\right|^{2} /\left\|\mathbf{a}_{1}\right\|^{2}<\alpha_{1,1}^{1}$ source . For the NC MUSIC-like algorithms, many numerical experiments for different arrays of sensors have shown that this factor is also strictly larger than 1, except for some specific phases for which it is equal to 1 .

Property 4: Finally, note that the phase of the correlation factor $\rho^{\prime}$, which corresponds to $\Delta \phi=\phi_{1}-\phi_{2}$ for rectilinear sources, can strongly impact the performance, through the term $\operatorname{Re}\left(\beta^{*} \rho^{\prime}\right)=\beta|| \rho^{\prime} \mid \cos \left(\angle \rho^{\prime}-\angle \beta\right)$ for the conventional MUSIC algorithm in (39) and (40) and through the terms $\widetilde{\beta}$ and $\frac{\tilde{\alpha}_{\phi, \phi}^{(k, k)}}{\tilde{\gamma}_{k}}$ for the NC MUSIC algorithm in (37), (38) and (41). We can clearly see that the asymptotic variances given for the conventional MUSIC algorithm in (39) and (40) are maximal [resp. minimal] for $\angle \rho^{\prime}=\angle \beta$ [resp. $\angle \rho^{\prime}= \pm \pi+\angle \beta$ ] with the associated largest and smallest asymptotic variances are obtained by replacing $\operatorname{Re}\left(\beta^{*} \rho^{\prime}\right)$ in (39) and (40) by $\operatorname{Re}\left(\beta^{*} \rho^{\prime}\right)=|\beta|\left|\rho^{\prime}\right|$ and $\operatorname{Re}\left(\beta^{*} \rho^{\prime}\right)=-|\beta|\left|\rho^{\prime}\right|$, respectively. In contrast the impact of the correlation phase $\Delta \phi$ is more difficult to analyze for the NC MUSIC algorithm due to the complicated expression of the phase and array geometry-dependent term $\frac{\tilde{\alpha}_{\phi, \phi}^{(k, k)}}{\tilde{\gamma}_{k}}$ in (37), (38) and (41).

Note that this sensitivity on the phase of the correlation seems to have been overlooked by the numerous performance analysis of subspace-based DOA estimation techniques such as conventional MUSIC algorithm for which this phase has always been assumed zero or $180^{\circ}$ (e.g., in [36]). Whereas, it is known [37] that the correlation phase has a strong effect on the associated Stochastic Cramér-Rao bound under certain conditions (small aperture arrays, large correlation magnitude and closely-spaced sources).

This phase assumed to be fixed during the array observation is a highly variable and unpredictable parameter in a multipath environment for which it is very sensitive to the difference between the propagation delays in the direct and secondary paths. Consequently the asymptotic variances may vary significantly from time to time and thus the performances are rather given by the mean of these asymptotic variances. For the conventional 
MUSIC algorithm (39) and (40) give:

$$
{\overline{\left[\mathbf{R}_{\mathrm{NG}}(\theta)\right]_{k, k}}}_{\overline{\left[\mathbf{R}_{\mathrm{G}}(\theta)\right]_{k, k}}}+(\eta-1) \frac{1}{\alpha_{k, k}}\left[\left(\frac{1+\left|\rho^{\prime}\right|^{2}}{\left(1-|\beta|^{2}\right)\left(1-\left|\rho^{\prime}\right|^{2}\right)^{2}}\right) \frac{1}{\left\|\mathbf{a}_{k}\right\|^{2}} \frac{1}{r^{2}}\right], \quad k=1,2,
$$

where

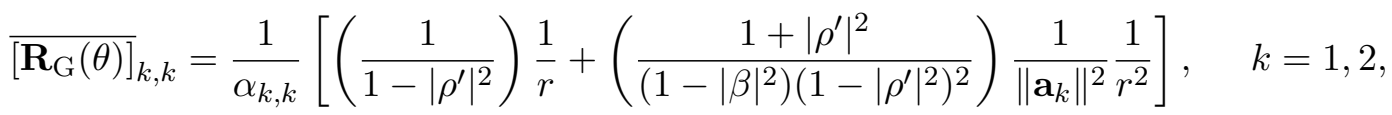

which now clearly monotonously increases with $\left|\rho^{\prime}\right|$. For the NC MUSIC algorithm, no closed-form expressions is attainable because of the complicated expression of $\frac{\tilde{\alpha}_{\phi, \phi}^{(k, k)}}{\tilde{\gamma}_{k}}$.

\section{NUMERICAL ILLUSTRATIONS}

This section illustrates the dependence of the provided asymptotic performance results in Subsection IV on geometric, phase and magnitude of sources correlation parameters and on the non-Gaussian distribution of the noise or of the observation by considering two illustration parts. Let us assume that $K=2$ narrowband equal-power rectilinear correlated signal sources with power $\sigma^{2}$ impinge on a uniform linear array of $N=6$ sensors $^{4}$ separated by a half-wavelength for which the steering vectors are $\mathbf{a}\left(\theta_{k}\right)=\left(1, e^{i \theta_{k}}, \ldots, e^{i(N-1) \theta_{k}}\right)^{T}$ where $\theta_{k}=\pi \sin \left(\omega_{k}\right), k=1,2$, with $\omega_{k}$ is the DOAs relative to the normal of array broadside. The phases $\phi_{k}, k=1,2$ associated with different propagation delays are assumed fixed, but unknown during the array observation and the performance depends only on $\Delta \phi \stackrel{\text { def }}{=}\left|\phi_{2}-\phi_{1}\right|$. The SNR is defined as $10 \log _{10}\left(\sigma_{s}^{2} / \sigma_{n}^{2}\right)$ dB. 1000 independent Monte Carlo runs have been performed where the number of snapshots is fixed at $T=500$ to obtain estimations of the mean squared error (MSE) $\mathrm{E}\left(\hat{\theta}_{1}-\theta_{1}\right)^{2}$.

In the first experiment, the noise $\mathbf{n}_{t}$ is either circular complex Student $t$-distributed with parameter $\nu>4$ to have finite fourth-order moment for which $\eta=\frac{\nu-2}{\nu-4}$ or C-CG distributed (obtained also for $\nu \rightarrow \infty$ ). We suppose the sources in model (1) consist of two multipaths issued from two independent BPSK modulated signals $e_{t, 1}$ and $e_{t, 2}$, for which we have $s_{t, 1}=e_{t, 1}$ and $s_{t, 2}=\rho e_{t, 1}+\sqrt{1-\rho^{2}} e_{t, 2}$. The two sources $s_{t, 1}$ and $s_{t, 2}$ are thus equal-powered with correlation $\rho$.

Fig. 1 compares the theoretical asymptotic variances of DOA estimates given by (37)-(38) and (39)-(40) associated respectively with SCM-based NC MUSIC algorithm (17) and SCM-based conventional MUSIC algorithm [26], and the corresponding MSEs for the two previously described noise models. It can be seen from this figure that the C-CES distributed noise model causes a deeper loss of performance of the SCM-based MUSIC algorithms for weak SNR and DOA separation. It may be observed, on the other hand, that the NC MUSIC SCM-based algorithms outperform the conventional MUSIC SCM-based algorithm in particular for low DOA separation as already shown in [8] for C-CG distributed noise. It can be seen also that the asymptotic

\footnotetext{
${ }^{4}$ except in Fig. 4 in which $N$ is variable.
} 
variances for the C-CES noise model coincide with the one for the C-CG noise model for sufficiently large values of SNR as predicted by Property 2. On the other hand, these figures confirm the agreement between the asymptotic variance and its corresponding MSE associated with both MUSIC SCM-based algorithms in a large domain of SNR and DOA separation, with a larger domain for the NC MUSIC SCM-based algorithm.

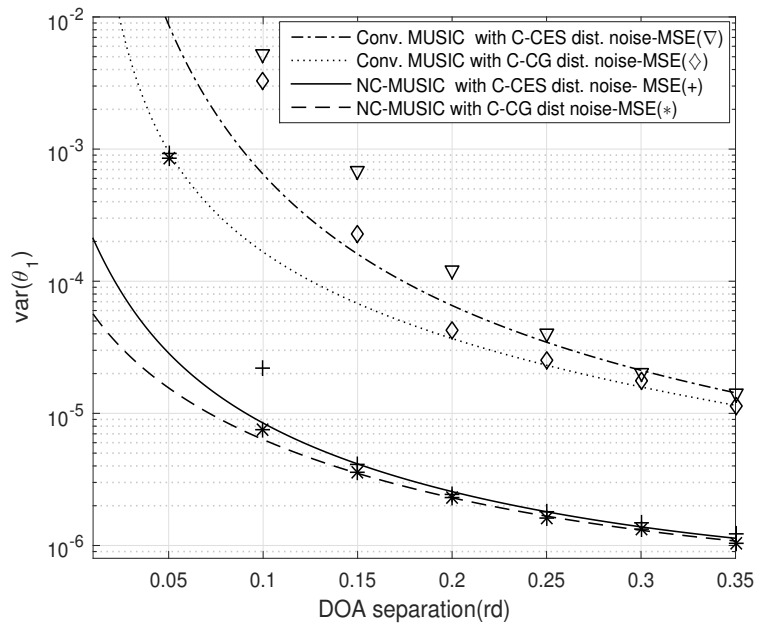

(a) $\mathrm{SNR}=20 d B$

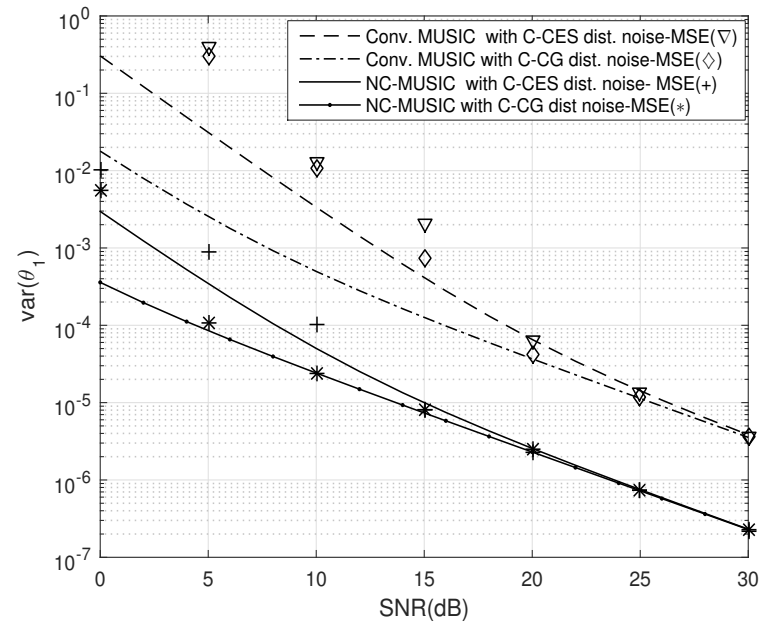

(b) $\Delta \theta=0.2 r d$

Fig. 1. Asymptotic variances $\operatorname{var}\left(\theta_{1, T}\right)$ given by (37)-(38) and (39)-(40) and its associated MSEs versus SNR (and versus DOA separation $\left.\Delta \theta=\left|\theta_{2}-\theta_{1}\right|\right)$ for C-CG and circular complex Student $t$-distributed noise models with $\nu=4.1$, fixed DOAs and phases with $\Delta \phi \stackrel{\text { def }}{=}\left|\phi_{2}-\phi_{1}\right|=0.1 r d$ and $\rho=\left|\rho^{\prime}\right|=0.5$.

Fig. 2 illustrates the behavior of the ratio between the asymptotic variances (37) and (38) respectively given for C-CG distributed noise and circular complex Student $t$-distributed noise as a function of SNR, correlation factor $\rho$, phase separation, and DOA separation and Student $t$-distribution noise parameter $\nu$. From Fig. 2(a)(b), it can be seen that the asymptotic variances are approximately equal for sufficiently high SNR, whereas the performance losses of SCM-based NC MUSIC algorithms become very prominent when SNR and DOA separation decrease and that $\rho$ increases. Fig. 2(c) exhibits the dependence of asymptotic variances on the phase separation $\Delta \phi$. It can be observed that the performance is very sensitive to $\Delta \phi$ for strongly correlated sources (i.e., $\rho \approx 1)$. Fig. $2(\mathrm{~d})$ shows that performance degradation is severe for small parameter $\nu(\nu \rightarrow 4)$, i.e., for heavy-tailed noise distributions and for strongly correlated sources (i.e., $\rho \approx 1$ ). Obviously, this ratio tends to 1 for $\nu \rightarrow \infty$ (C-CG noise model).

Fig. 3 illustrates property 2 which shows that for $\Delta \theta=0.002 r d$ and $\Delta \phi=0.02 r d$ associated with $\tilde{\beta}=0.9997$ and $\beta=1.0000+0.0049 i$, the asymptotic variances are not necessarily increasing functions of $|\rho|$. Finally, Fig. 4 examines the strong dependence of the performance of the conventional MUSIC algorithm on the phase of the correlation, by plotting the corresponding asymptotic variance given by (40) for C-CG distributed observations as a function of $\angle \rho^{\prime}$ for different values of $\Delta \theta$ with $\left|\rho^{\prime}\right|=0.95$. To better understand the effect of $\angle \rho^{\prime}$, we also 


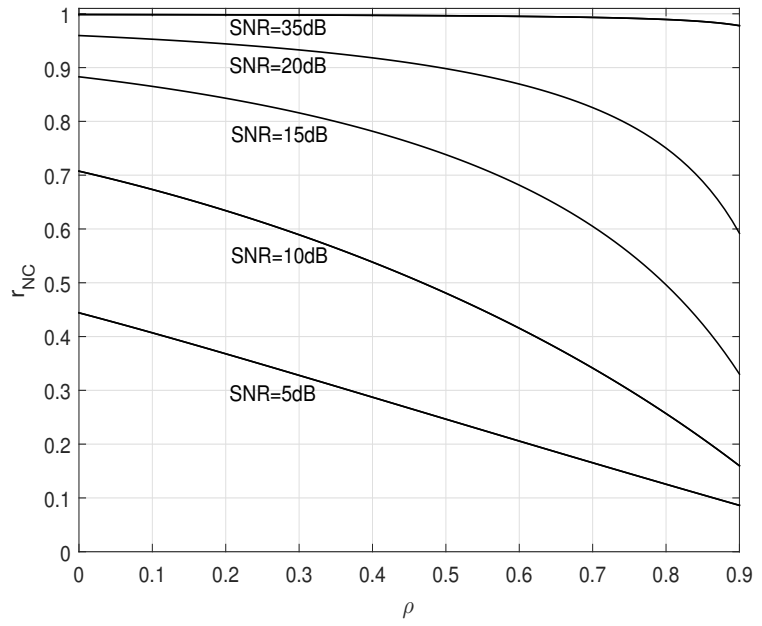

(a) $\Delta \theta=0.2 r d, \Delta \phi=0.1 r d, \nu=4.1$

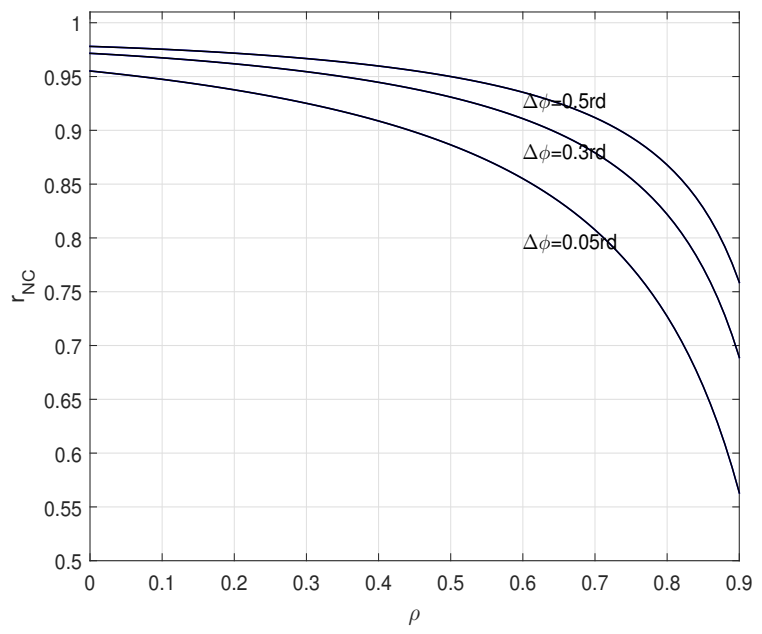

(c) $\mathrm{SNR}=20 d B, \Delta \theta=0.2 r d, \nu=4.1$

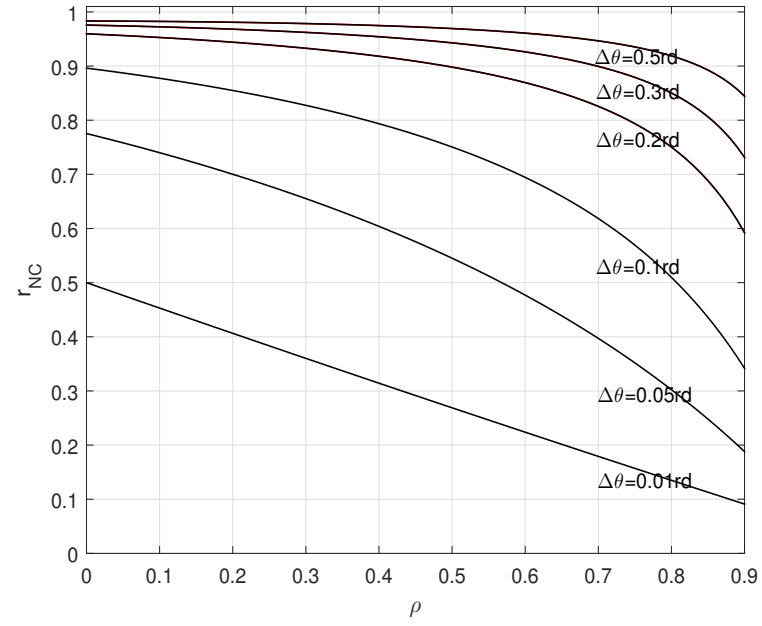

(b) $\mathrm{SNR}=20 d B, \Delta \phi=0.1 r d, \nu=4.1$

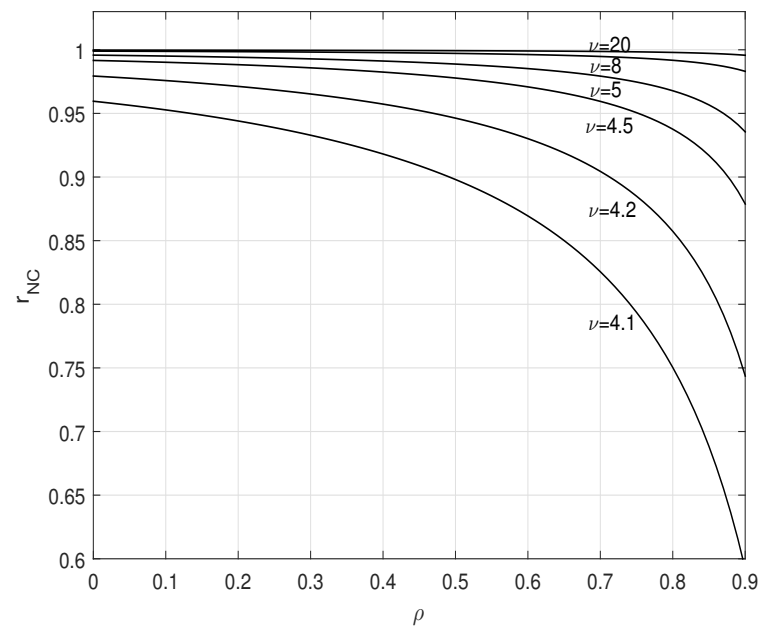

(d) $\mathrm{SNR}=20 d B, \Delta \theta=0.2 r d, \Delta \phi=0.1 r d$,

Fig. 2. Ratio between (37) and (38) (denoted by $r_{\mathrm{NC}} \stackrel{\text { def }}{=} R_{\mathrm{G}}^{\mathrm{NC}}\left(\theta_{1}\right) / R_{\mathrm{NG}}^{\mathrm{NC}}\left(\theta_{1}\right)$ ) as a function of $\rho$ for different values of SNR, $\Delta \theta$, $\Delta \phi$ and $\nu$.

plot the associated stochastic Cramér-Rao bound (CRB) derived in [35] for C-CG distributed observations. It can be clearly seen, as predicted by Property 4, that the asymptotic variance of the conventional MUSIC algorithm which depends on the geometric phase term $\angle \beta=(N-1) \Delta \theta / 2$ is maximal [resp., minimal] for $\angle \rho^{\prime}=\angle \beta$ [resp., $\angle \rho^{\prime}=\angle \beta+\pi$ ] and consequently the curses are shifted to the right when $\Delta \theta$ increases. Furthermore, we note that the corresponding largest and smallest asymptotic variances respectively remain closer to the CRB. Under these conditions, the conventional MUSIC algorithm is asymptotically efficient despite a very strong correlation, whereas this algorithm has always been considered inefficient for strongly correlated sources and a low number of sensors (see e.g., [35]). The corresponding asymptotic variance of MUSIC and CRB obviously increase when $\Delta \theta$ decreases and note that the correlation phase strongly affects the CRB compared to the 


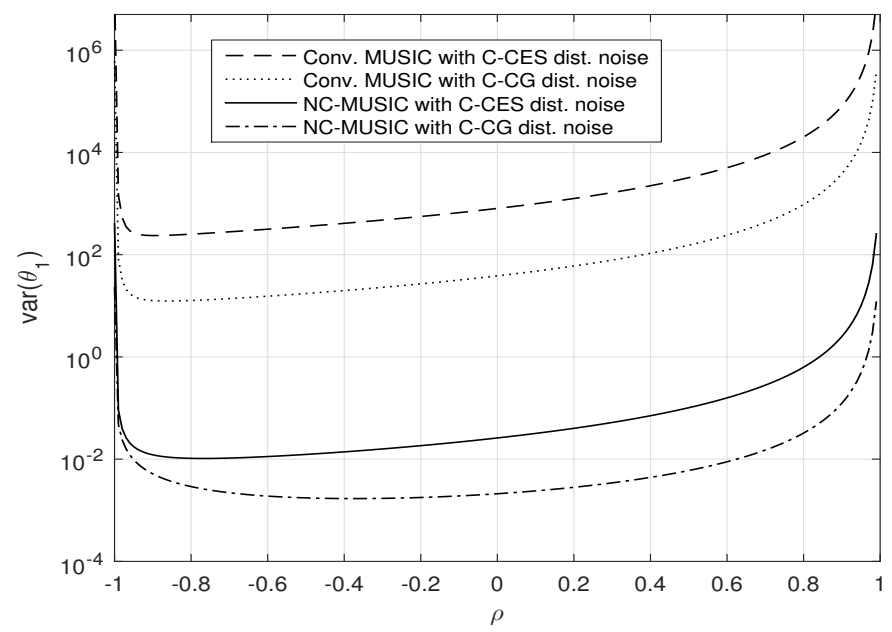

Fig. 3. Asymptotic variances $\operatorname{var}\left(\theta_{1, T}\right)$ associated with conventional MUSIC [resp., NC MUSIC] algorithm given by (40) [resp., by (38)] for C-CG noise model, and by (39) [resp., (37)] for circular complex Student $t$-distributed noise model with $\nu=4$.1 and $\mathrm{SNR}=20 d B$.

impact on the corresponding asymptotic variance of MUSIC algorithm which is lower.

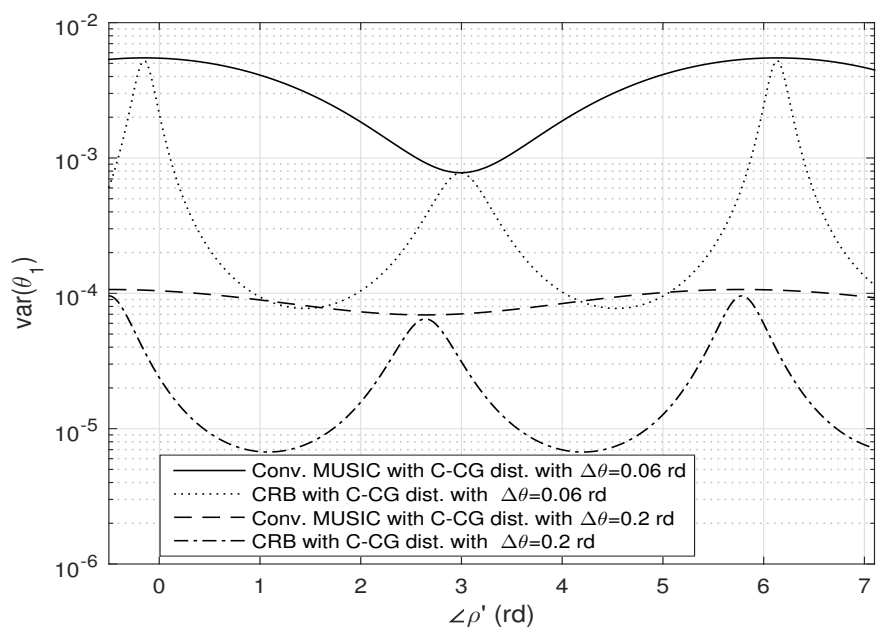

Fig. 4. Asymptotic variance $\operatorname{var}\left(\theta_{1, T}\right)$ associated with the conventional MUSIC algorithm given by (40) for C-CG distributed observations, compared to the stochastic CRB, for different values of $\Delta \theta$ with $\left|\rho^{\prime}\right|=0.95, \mathrm{SNR}=20 d B$ and $T=2000$.

In this second experiment, we assume that the observations $\mathbf{y}_{t}$ follow either a NC complex Student $t$ distribution with parameter $\nu>4$, which has heavier tails than the Gaussian, or a NC complex generalized Gaussian distribution with exponent $\zeta>0$ for which $\vartheta_{1, \mathrm{ML}}=\frac{N+1}{N+\zeta}$ and $\vartheta_{1, \mathrm{SCM}}=\eta=\frac{N}{N+1} \frac{\Gamma(N / \zeta) \Gamma((N+2) / \zeta)}{\Gamma((N+1) / \zeta)^{2}}$ [26] with $\zeta=1, \zeta<1$ and $\zeta>1$ referring respectively to the NC-CG distribution, to NC super-Gaussian and NC sub-Gaussian distributions. These distributions have a structured extended covariance matrix $\mathbf{R}_{\tilde{y}}$ given by (7), for which the robustness of the non-circular subspace-based DOA estimation algorithms using robust covariance matrix estimators is evaluated. 


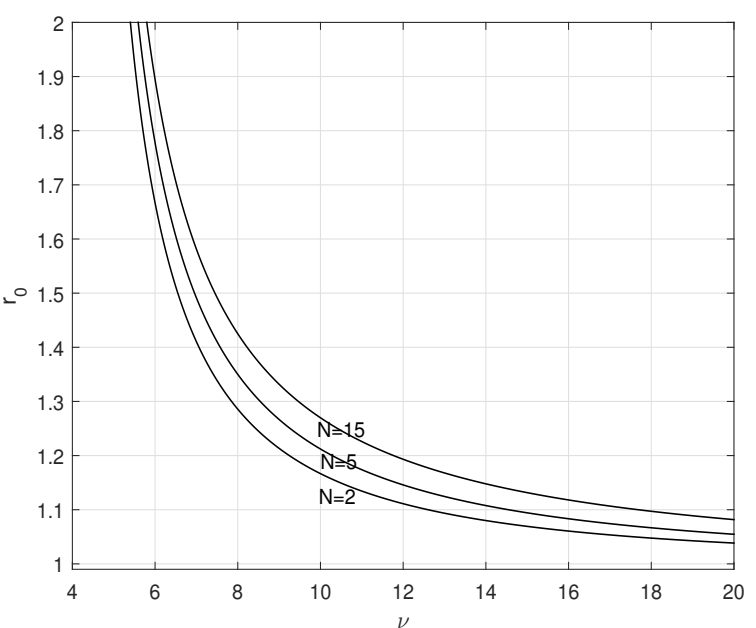

(a) $r_{0}=\vartheta_{1, \mathrm{SCM}} / \vartheta_{1, \mathrm{ML}}$

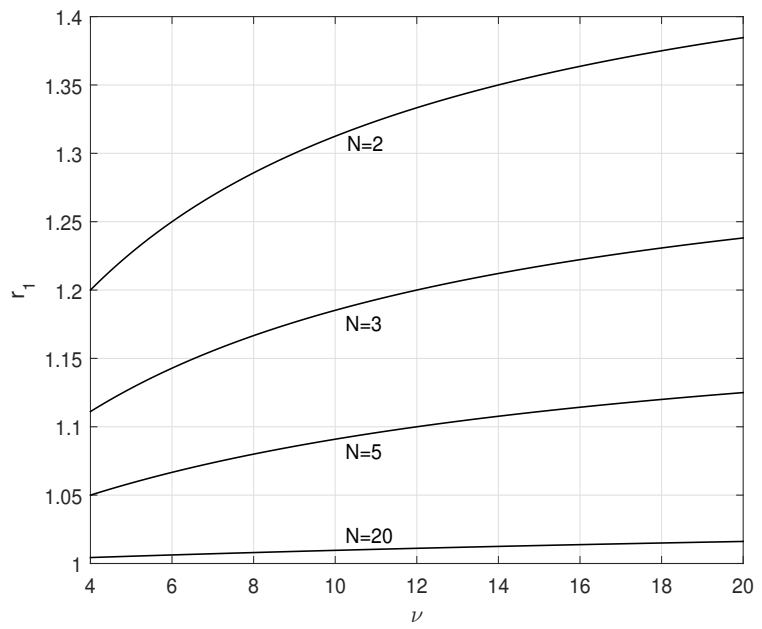

(c) $r_{1}=\vartheta_{1, \text { Tyler }} / \vartheta_{1, \mathrm{ML}}$

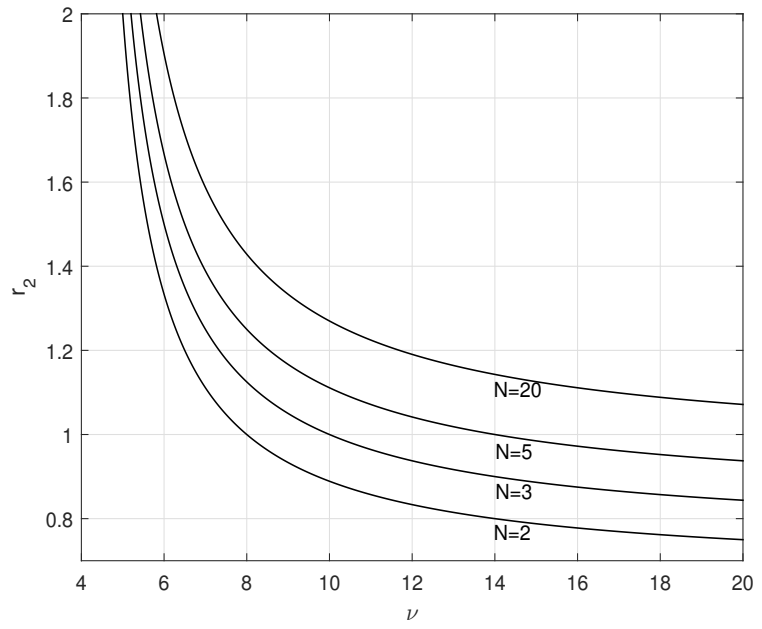

(e) $r_{2}=\vartheta_{1, \mathrm{SCM}} / \vartheta_{1, \text { Tyler }}$

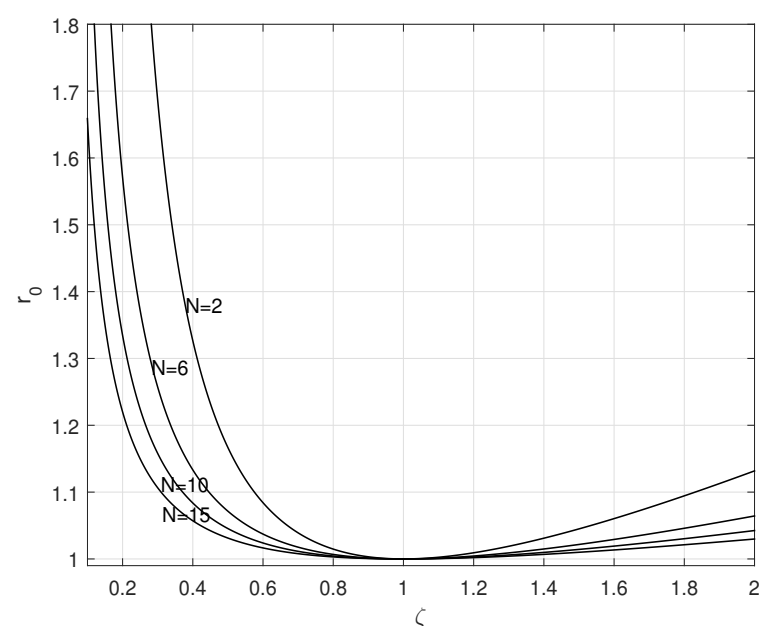

(b) $r_{0}=\vartheta_{1, \mathrm{SCM}} / \vartheta_{1, \mathrm{ML}}$

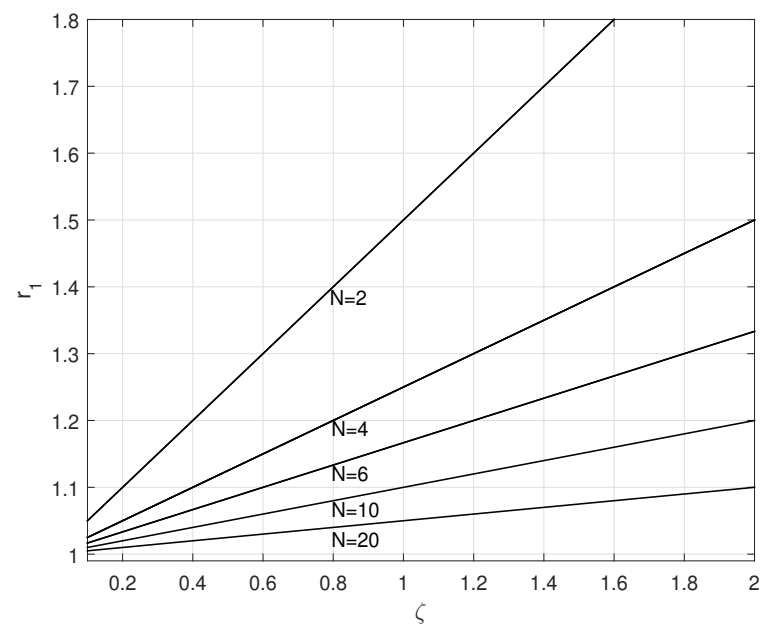

(d) $r_{1}=\vartheta_{1, \text { Tyler }} / \vartheta_{1, \mathrm{ML}}$

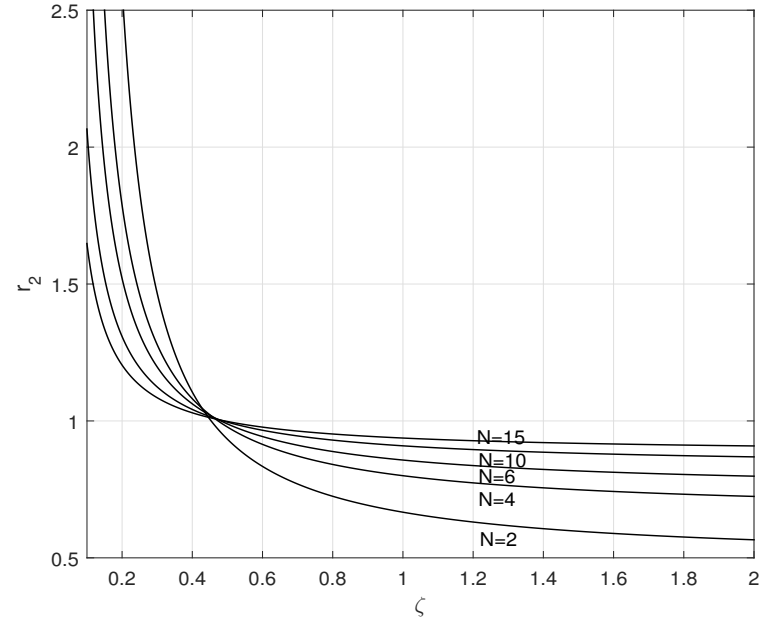

(f) $r_{2}=\vartheta_{1, \mathrm{SCM}} / \vartheta_{1, \text { Tyler }}$

Fig. 5. Ratios $r_{0}=\vartheta_{1, \mathrm{SCM}} / \vartheta_{1, \mathrm{ML}}, r_{1}=\vartheta_{1, \text { Tyler }} / \vartheta_{1, \mathrm{ML}}$ and $r_{2}=\vartheta_{1, \mathrm{SCM}} / \vartheta_{1 \text {,Tyler }}$ versus NC complex Student $t$-distribution parameter $\nu$ (first column) and versus NC complex generalized Gaussian distribution parameter $\zeta$ (second column) for different values of $N$. 
To supplement our discussion below Result 4, Fig. 5 illustrates the robustness of NC MUSIC DOA estimation algorithms based on the ML $M$-estimator with respect to the ones based on Tyler's $M$-estimator and SCM estimator, by plotting the ratios $r_{0} \stackrel{\text { def }}{=}\left[\mathbf{R}_{\mathrm{NC}-\mathrm{CES}}^{\mathrm{SCM}}(\theta)\right]_{1,1} /\left[\mathbf{R}_{\mathrm{NC}-\mathrm{CES}}^{\mathrm{ML}}(\theta)\right]_{1,1}=\vartheta_{1, \mathrm{SCM}} / \vartheta_{1, \mathrm{ML}}$, $r_{1} \stackrel{\text { def }}{=}\left[\mathbf{R}_{\mathrm{NC}-\mathrm{CES}}^{\text {Tyler }}(\theta)\right]_{1,1} /\left[\mathbf{R}_{\mathrm{NC}-\mathrm{CES}}^{\mathrm{ML}}(\theta)\right]_{1,1}=\vartheta_{1, \text { Tyler }} / \vartheta_{1, \mathrm{ML}}$ and $r_{2} \stackrel{\text { def }}{=}\left[\mathbf{R}_{\mathrm{NC}-\mathrm{CES}}^{\mathrm{SCM}}(\theta)\right]_{1,1} /\left[\mathbf{R}_{\mathrm{NC}-\mathrm{CES}}^{\text {Tyler }}(\theta)\right]_{1,1}=$ $\vartheta_{1, \mathrm{SCM}} / \vartheta_{1, \text { Tyler }}$ for different values of $N$, versus $\nu$ and $\zeta$, respectively. From Fig. 5(a)-(c) and (b)-(d), we observe that NC MUSIC DOA estimation algorithm based on ML $M$-estimator outperforms the ones based on the SCM estimator and Tyler's $M$-estimator as predicted by Result 4 , but both ratios $r_{1}$ tend to 1 when $N \rightarrow \infty$ and therefore, the NC MUSIC DOA estimation algorithms based on the ML $M$-estimator provided similar performance as the ones based on Tyler's $M$-estimator as $N$ increases. For small values of $N$, both ratios $r_{0}$ increase when the distribution moves away from the Gaussian distribution, i.e., for $\nu$ decreasing and $\zeta$ moving away from 1. As a result, the performance of the NC MUSIC DOA estimation algorithms based on the SCM degrade when the distributions move away from the Gaussian distribution. By contrast, both ratios $r_{1}$ increase when $\nu$ and $\zeta$ increase, being very large only for large $\zeta$ (i.e., for light-tailed distributions). From Fig. 5(e) and (f), we see that the NC MUSIC DOA estimation algorithms based on Tyler's $M$-estimator have much better performance than the ones based on SCM for heavy-tailed distributions (i.e., for $\nu$ close to 4 and small values of $\zeta$ ), whereas the performance is poorly degraded for the Gaussian distribution (i.e., for $\nu=\infty, \zeta=1$ ). But note that the Tyler's $M$-estimator significantly degrades the performance for strongly light-tailed distributions (i.e., for $\zeta \gg 1$ ). Fig. 6 compares the NC stochastic CRB derived in [25] and circular stochastic CRB derived in [39] and [40], to the asymptotic variances of DOA estimates (41) and [26, Eq.(34)] obtained, respectively, with the NC MUSIC algorithm (17) and conventional MUSIC algorithm based on SCM estimator (i.e., $\vartheta_{1}=\vartheta_{1, \mathrm{SCM}}=\eta$ ) and ML $M$-estimator (i.e., $\vartheta_{1}=\vartheta_{1, \mathrm{ML}}$ ), and the corresponding MSEs. From this figure, we can observe the good agreement between the theoretical asymptotic variance associated with the NC MUSIC algorithm and its corresponding MSE for a wide range of SNR values, whereas the theoretical asymptotic variance associated with conventional MUSIC algorithm and its corresponding MSE are in good agreement only at high SNR. Here again, it can be noted that the performance of the NC MUSIC algorithms exploiting the non-circularity property of the observations outperforms those of the conventional MUSIC algorithm build only from a SCM of (25). It can also be observed that the NC MUSIC algorithms based on $M$-estimate are asymptotically efficient compared to the CRB for a wide range of SNR values, which is not the case for the conventional MUSIC algorithm which is asymptotically efficient at high SNR.

\section{CONCLUSION}

This paper has shown that all the NC subspace-based algorithms built from the SCM designed for uncorrelated rectilinear sources embedded in spatially white C-CG noise can be also applied for correlated rectilinear sources 


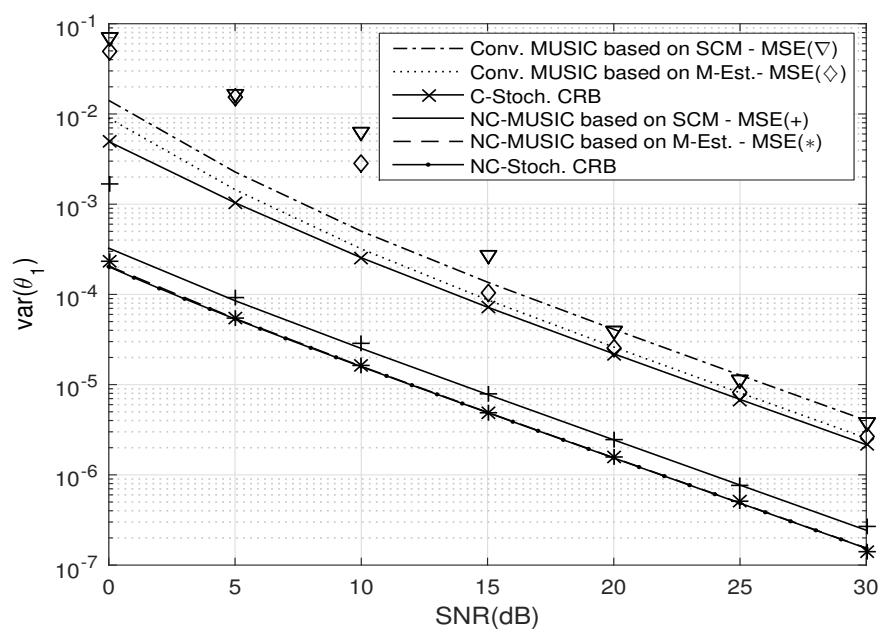

Fig. 6. Circular and NC stochastic CRBs, asymptotic variances given by (29) and [26, Eq.(34)] and associated MSEs versus SNR for NC complex generalized Gaussian distributed observations with exponent $\zeta=0.2$, fixed DOAs and phases with $\Delta \theta=0.25(r d)$ and $\Delta \phi=0.2(r d)$, and $\rho=\left|\rho^{\prime}\right|=0.5$.

in the contexts of SCM estimate with C-CES noise and $M$-estimate with NC-CES observations. A perturbation analysis has been performed to derive closed-form expressions for the asymptotic covariance matrices of DOA estimates for three NC MUSIC-like algorithms in two CES data models. Interpretable closed-form expressions of the asymptotic variance of the estimated DOA of two equi-power correlated sources has been derived for the first time. A number of properties that highlight how the asymptotic variances of NC MUSIC-like DOA estimation algorithms depend on key parameters such as SNR, DOA, phase and magnitude of the correlation and C-CES noise parameters were derived. These results were compared with those of the conventional MUSIC DOA estimation algorithm, and a significant gain was quantified for relatively small DOA separation when using non-circular signals. Analytical robustness results were illustrated via several numerical examples using robust covariance matrix estimators instead of the SCM, proving that the use of robust $M$-estimators enhances the robustness of the subspace-based DOA estimation algorithms against heavy-tailed NC-CES observations model deviations, with negligible loss in performance for NC-CG distributed observations. Finally, we note that the presented methodology also applies to the asymptotic performance analysis of the NC ESPRIT-like algorithms in the contexts of SCM estimates with NC deterministic or stochastic sources embedded in C-CES noise and $M$-estimates with NC-CES observations.

\section{APPENDIX}

Proof of Result 1: Using the central limit theorem applied to the independent identically distributed complex r.v. $\operatorname{vec}\left(\tilde{\mathbf{y}}_{t} \tilde{\mathbf{y}}_{t}^{H}\right)=\tilde{\mathbf{y}}_{t}^{*} \otimes \tilde{\mathbf{y}}_{t}$, the sequence $\sqrt{T}\left(\operatorname{vec}\left(\mathbf{R}_{\tilde{y}, T}\right)-\operatorname{vec}\left(\mathbf{R}_{\tilde{y}}\right)\right)$ is asymptotically zero-mean Gaussian distributed with covariance $\mathbf{R}_{r_{\tilde{y}}}$ and complementary covariance $\mathbf{C}_{r_{\tilde{y}}}=\mathbf{R}_{r_{\tilde{y}}} \mathbf{K}$. Thanks to simple algebraic 
manipulations, we obtain

$$
\begin{aligned}
\mathbf{R}_{r_{\tilde{y}}} & =\left(\widetilde{\mathbf{A}}^{*} \otimes \widetilde{\mathbf{A}}\right) \mathbf{R}_{r_{s}}\left(\widetilde{\mathbf{A}}^{T} \otimes \widetilde{\mathbf{A}}^{H}\right)+\left(\widetilde{\mathbf{A}}^{*} \mathbf{R}_{s} \widetilde{\mathbf{A}}^{T}\right) \otimes \mathbf{R}_{\tilde{n}}+\mathbf{R}_{\tilde{n}}^{*} \otimes\left(\widetilde{\mathbf{A}} \mathbf{R}_{s} \widetilde{\mathbf{A}}^{H}\right) \\
& +\mathbf{K}\left[\left(\widetilde{\mathbf{A}} \mathbf{R}_{s} \widetilde{\mathbf{A}}^{T}\right) \otimes\left(\mathbf{J} \mathbf{R}_{\tilde{n}}\right)+\left(\mathbf{J} \mathbf{R}_{\tilde{n}}^{*}\right) \otimes\left(\widetilde{\mathbf{A}}^{*} \mathbf{R}_{s} \widetilde{\mathbf{A}}^{H}\right)\right]+\mathbf{R}_{r_{\tilde{n}}}
\end{aligned}
$$

where $\mathbf{R}_{\tilde{n}}=\sigma_{n}^{2} \mathbf{I}, \mathbf{R}_{r_{s}}$ is the covariance of $\mathbf{s}_{t} \otimes \mathbf{s}_{t}$ (which is zero in the deterministic model) and $\mathbf{R}_{r_{\tilde{n}}}$ is the covariance of $\tilde{\mathbf{n}}_{t}^{*} \otimes \tilde{\mathbf{n}}_{t}$, which is simplified for both C-CES or C-CCG distributed $\mathbf{n}_{t}$ (3) through simple algebraic manipulations as:

$$
\mathbf{R}_{r_{\tilde{n}}}=\sigma_{n}^{4}\left\{(\mathbf{I} \otimes \mathbf{I})+\mathbf{K}(\mathbf{J} \otimes \mathbf{J})+(\eta-1)\left[(\mathbf{I} \otimes \mathbf{I})+\mathbf{K}(\mathbf{J} \otimes \mathbf{J})+\operatorname{vec}(\mathbf{I}) \operatorname{vec}^{T}(\mathbf{I})\right]\right\} .
$$

Using the standard theorem of continuity (see e.g., [41, p.122]) on regular functions of asymptotically Gaussian statistics applied to the mapping $\mathbf{R}_{\tilde{y}, T} \longmapsto \Pi_{\tilde{y}, T}$, we obtain similarly to [8, Th.3], that the sequence $\sqrt{T}\left(\operatorname{vec}\left(\boldsymbol{\Pi}_{\tilde{y}, T}\right)-\operatorname{vec}\left(\boldsymbol{\Pi}_{\tilde{y}}\right)\right)$ converges to a zero-mean Gaussian distribution with covariance $\mathbf{R}_{\pi_{\tilde{y}}}$ and complementary covariance $\mathbf{C}_{\pi_{\tilde{y}}}=\mathbf{R}_{\pi_{\tilde{y}}} \mathbf{K}$ given by

$$
\begin{aligned}
\mathbf{R}_{\pi_{\tilde{y}}} & =(\mathbf{I}+\mathbf{K}(\mathbf{J} \otimes \mathbf{J}))\left[\left(\tilde{\mathbf{U}}^{T} \otimes \boldsymbol{\Pi}_{\tilde{y}}\right)+\left(\boldsymbol{\Pi}_{\tilde{y}}^{T} \otimes \widetilde{\mathbf{U}}\right)\right. \\
& +(\eta-1)(\mathbf{I}+\mathbf{K}(\mathbf{J} \otimes \mathbf{J}))\left[\left(\tilde{\mathbf{U}}^{\prime} T \otimes \boldsymbol{\Pi}_{\tilde{y}}\right)+\left(\boldsymbol{\Pi}_{\tilde{y}}^{T} \otimes \widetilde{\mathbf{U}}^{\prime}\right) .\right.
\end{aligned}
$$

Then applying again the standard theorem of continuity to the mapping (8) $\Pi_{\tilde{y}, T} \stackrel{\operatorname{alg}}{\longmapsto} \widehat{\boldsymbol{\theta}}_{T}$, we obtain similarly to [8, Th.5], that the sequence of the DOA estimates $\sqrt{T}\left(\widehat{\boldsymbol{\theta}}_{T}-\boldsymbol{\theta}\right)$ asymptotically converges to a zero-mean Gaussian distribution, whose covariance is deduced and Result 1 is proved thanks to algebraic manipulations similar to those developped in [8] and [26].

Proof of Result 3: The proof is based on the asymptotic distribution of $\operatorname{vec}\left(\boldsymbol{\Pi}_{\tilde{y}, T}\right)$ associated with the $M$ estimate $\boldsymbol{\Gamma}_{\tilde{y}, T}^{u}$. In $[29, \operatorname{Res} .1]$, it is proved that the sequence $\sqrt{T}\left(\operatorname{vec}\left(\boldsymbol{\Pi}_{\tilde{y}, T}\right)-\operatorname{vec}\left(\boldsymbol{\Pi}_{\tilde{y}}\right)\right)$ is asymptotically zero-mean Gaussian distributed with covariance $\mathbf{R}_{r_{\tilde{y}}}$ and complementary covariance $\mathbf{C}_{r_{\tilde{y}}}=\mathbf{R}_{r_{\tilde{y}}} \mathbf{K}$ given by

$$
\mathbf{R}_{\pi_{\tilde{y}}}=\frac{\vartheta_{1}}{\sigma_{u}^{2}}(\mathbf{I}+\mathbf{K}(\mathbf{J} \otimes \mathbf{J}))\left[\left(\widetilde{\mathbf{U}}^{T} \otimes \boldsymbol{\Pi}_{\tilde{y}}\right)+\left(\boldsymbol{\Pi}_{\tilde{y}}^{T} \otimes \widetilde{\mathbf{U}}\right)\right]
$$

where $\vartheta_{1}$ is given by (30) and $\sigma_{u}$ is solution of (15). Applying again the standard theorem of continuity to the mapping (8), $\boldsymbol{\Pi}_{\tilde{y}, T} \stackrel{\text { alg }}{\longmapsto} \widehat{\boldsymbol{\theta}}_{T}$, the first part of Result 3 is proved.

For the ML $M$-estimates $\mathrm{E}\left[\psi\left(\mathcal{Q}_{t}\right) \mathcal{Q}_{t}\right]=N$ from (13) and (11) and thus $\sigma_{u}=1$ from (15). Consequently, (30) reduces to

$$
\vartheta_{1}=\frac{N(N+1) \mathrm{E}\left[\psi^{2}\left(\mathcal{Q}_{t}\right) \mathcal{Q}_{t}^{2}\right]}{\left(N(N+1)+\mathrm{E}\left[\psi^{\prime}\left(\mathcal{Q}_{t}\right) \mathcal{Q}_{t}^{2}\right]\right)^{2}}
$$


Using the p.d.f. (9) of the r.v. $\mathcal{Q}_{t}$, we straightforwardly get:

$$
\mathrm{E}\left[\psi^{2}\left(\mathcal{Q}_{t}\right) \mathcal{Q}_{t}^{2}\right]-\mathrm{E}\left[\psi^{\prime}\left(\mathcal{Q}_{t}\right) \mathcal{Q}_{t}^{2}\right]=\int_{0}^{\infty} \delta_{N, g}^{-1} q^{N+1} \frac{d^{2} g(q)}{d q^{2}} d q
$$

where

$$
\int_{0}^{\infty} \delta_{N, g}^{-1} q^{N+1} \frac{d^{2} g(q)}{d q^{2}} d q=\left[\delta_{N, g}^{-1} q^{N+1} \frac{d g(q)}{d q}\right]_{0}^{\infty}-(N+1) \int_{0}^{\infty} \delta_{N, g}^{-1} q^{N} \frac{d g(q)}{d q} d q
$$

The second term can be simplified as follows

$$
\int_{0}^{\infty} \delta_{N, g}^{-1} q^{N} \frac{d g(q)}{d q} d q=\left[\delta_{N, g}^{-1} q^{N} g(q)\right]_{0}^{\infty}-N \int_{0}^{\infty} \delta_{N, g}^{-1} q^{N-1} g(q) d q=-N,
$$

because $\lim _{q \rightarrow \infty} q^{N+1} \frac{d g(q)}{d q}=\lim _{q \rightarrow \infty} q^{N} g(q)=0$ using the fact that the fourth-order moment of $\mathcal{Q}_{t}$ is assumed finite and $\int_{0}^{\infty} \delta_{N, g}^{-1} q^{N-1} g(q) d q=1$. Hence, $\mathrm{E}\left[\psi^{2}\left(\mathcal{Q}_{t}\right) \mathcal{Q}_{t}^{2}\right]=N(N+1)+\mathrm{E}\left[\psi^{\prime}\left(\mathcal{Q}_{t}\right) \mathcal{Q}_{t}^{2}\right]$, and using (49), (31) is proved.

Because the extended SCM is the $M$-estimate associated with $u(t)=1$ for which $c_{u}=0$ and $\sigma_{u}=1$, and thus $\vartheta_{1}=\eta$ from (4) and (30).

Proof of Result 4: First note that using the p.d.f. (11) of the r.v. $\mathcal{Q}_{t}$, we get

$$
\begin{aligned}
\mathrm{E}\left[\psi\left(\mathcal{Q}_{t}\right) \mathcal{Q}_{t}^{2}\right] & =-\int_{0}^{\infty} \delta_{N, g}^{-1} q^{N+1} \frac{d g(q)}{d q} d q \\
& =-\left[\delta_{N, g}^{-1} q^{N+1} g(q)\right]_{0}^{\infty}+(N+1) \int_{0}^{\infty} \delta_{N, g}^{-1} q^{N} g(q) d q \\
& =(N+1) \mathrm{E}\left(\mathcal{Q}_{t}\right)=N(N+1),
\end{aligned}
$$

because $\lim _{q \rightarrow \infty} q^{N+1} g(q)=0$ using the fact that the fourth-order moment of $\mathcal{Q}_{t}$ is assumed finite and $\mathrm{E}\left(\mathcal{Q}_{t}\right)=N$. The Cauchy-Schwarz inequality yields

$$
N^{2}(N+1)^{2}=\left(\mathrm{E}\left[\psi\left(\mathcal{Q}_{t}\right) \mathcal{Q}_{t}^{2}\right]\right)^{2} \leq \mathrm{E}\left(\mathcal{Q}_{t}^{2}\right) \mathrm{E}\left[\psi^{2}\left(\mathcal{Q}_{t}\right) \mathcal{Q}_{t}^{2}\right]
$$

and thus

$$
\vartheta_{1, \mathrm{ML}}=\frac{N(N+1)}{\mathrm{E}\left[\psi^{2}\left(\mathcal{Q}_{t}\right) \mathcal{Q}_{t}^{2}\right]} \leq \frac{\mathrm{E}\left(\mathcal{Q}_{t}^{2}\right)}{N(N+1)}=\eta
$$

The proof of (35) follows immediately from the Cauchy-Schwarz inequality which gives $\mathrm{E}\left[\left(\psi\left(\mathcal{Q}_{t}\right) \mathcal{Q}_{t}\right)^{2}\right] \geq$ $\left(\mathrm{E}\left[\psi\left(\mathcal{Q}_{t}\right) \mathcal{Q}_{t}\right]\right)^{2}=N^{2}$ using $\mathrm{E}\left(\psi\left(\mathcal{Q}_{t}\right) \mathcal{Q}_{t}\right)=N$ from $(15)$.

Proof of Result 5: To give the expression of $\widetilde{\mathbf{U}}$ and $\widetilde{\mathbf{U}}^{\prime}$ in (23) for two equi-powered sources, amounts to deriving the two non-zero eigenvalues and the associated eigenvectors of the rank two matrix $\widetilde{\mathbf{S}}=$ $\sigma_{s}^{2}\left(\widetilde{\mathbf{a}}_{1}, \widetilde{\mathbf{a}}_{2}\right)\left(\begin{array}{c}\widetilde{\mathbf{a}}_{1}^{H}+\rho \widetilde{\mathbf{a}}_{2}^{H} \\ \rho \widetilde{\mathbf{a}}_{1}^{H}+\widetilde{\mathbf{a}}_{2}^{H}\end{array}\right)$. The non-zero eigenvalues of $\widetilde{\mathbf{S}}$ are derived from the roots of the quadratic polynomial: 
$\lambda^{2}-\operatorname{Tr}(\widetilde{\mathbf{S}}) \lambda+\operatorname{det}\left[\sigma_{s}^{2}\left(\begin{array}{c}\widetilde{\mathbf{a}}_{1}^{H}+\rho \widetilde{\mathbf{a}}_{2}^{H} \\ \rho \widetilde{\mathbf{a}}_{1}^{H}+\widetilde{\mathbf{a}}_{2}^{H}\end{array}\right)\left(\widetilde{\mathbf{a}}_{1}, \widetilde{\mathbf{a}}_{2}\right)\right]$, which give the eigenvalues $\lambda_{1}=2\left\|\mathbf{a}_{k}\right\|^{2} \sigma_{s}^{2}(1-\beta)(1-\rho)$ and $\lambda_{2}=2\left\|\mathbf{a}_{k}\right\|^{2} \sigma_{s}^{2}(1+\beta)(1+\rho)$. Associated eigenvectors are $\mathbf{v}_{1}=\widetilde{\mathbf{a}}_{1}-\widetilde{\mathbf{a}}_{2}$ and $\mathbf{v}_{2}=\widetilde{\mathbf{a}}_{1}+\widetilde{\mathbf{a}}_{2}$. This allows us to deduce the expressions of $\widetilde{\mathbf{U}}=\left(\frac{\sigma_{n}^{2}}{\lambda_{1}}+\frac{\sigma_{n}^{4}}{\lambda_{1}^{2}}\right) \frac{\mathbf{v}_{1} \mathbf{v}_{1}^{H}}{\left\|\mathbf{v}_{1}\right\|^{2}}+\left(\frac{\sigma_{n}^{2}}{\lambda_{2}}+\frac{\sigma_{n}^{4}}{\lambda_{2}^{2}}\right) \frac{\mathbf{v}_{2} \mathbf{v}_{2}^{H}}{\left\|\mathbf{v}_{2}\right\|^{2}}$ and $\widetilde{\mathbf{U}}^{\prime}=\frac{\sigma_{n}^{4}}{\lambda_{1}^{2}} \frac{\mathbf{v}_{1} \mathbf{v}_{1}^{H}}{\left\|\mathbf{v}_{1}\right\|^{2}}+\frac{\sigma_{n}^{4}}{\lambda_{2}^{2}} \frac{\mathbf{v}_{2} \mathbf{v}_{2}^{H}}{\left\|\mathbf{v}_{2}\right\|^{2}}$ and then of $\widetilde{\mathbf{a}}_{k}^{H} \widetilde{\mathbf{U}} \widetilde{\mathbf{a}}_{k}$ and $\widetilde{\mathbf{a}}_{k}^{H} \widetilde{\mathbf{U}}^{\prime} \widetilde{\mathbf{a}}_{k}$, w.r.t.. the parameters $\sigma_{s}^{2}, \sigma_{n}^{2}, \rho$ and $\beta$. Plugging these expressions into (23) proves Result 5.

Proof of Result 6: The expression of $\mathbf{U}$ and $\mathbf{U}^{\prime}$ in (27) for two equi-powered sources are also derived from the two non-zero eigenvalues and the associated eigenvectors of the rank two matrix $\mathbf{S}=$ $\sigma_{s}^{2}\left(\mathbf{a}_{1}, \mathbf{a}_{2}\right)\left(\begin{array}{c}\mathbf{a}_{1}^{H}+\rho^{\prime} \mathbf{a}_{2}^{H} \\ \rho^{\prime *} \mathbf{a}_{1}^{H}+\mathbf{a}_{2}^{H}\end{array}\right)$. The non-zero eigenvalues of $\mathbf{S}$ are also the roots of the quadratic polynomial: $\lambda^{2}-\operatorname{Tr}(\mathbf{S}) \lambda+\operatorname{det}\left[\sigma_{s}^{2}\left(\begin{array}{c}\mathbf{a}_{1}^{H}+\rho^{\prime} \mathbf{a}_{2}^{H} \\ \rho^{\prime} \mathbf{a}_{1}^{H}+\mathbf{a}_{2}^{H}\end{array}\right)\left(\mathbf{a}_{1}, \mathbf{a}_{2}\right)\right]$ which are $\lambda_{k}=\left\|\mathbf{a}_{k}\right\|^{2} \sigma_{s}^{2}\left(\alpha \pm \sqrt{\alpha^{2}-\gamma}\right), k=1,2$, with $\alpha \stackrel{\text { def }}{=}$ $1+\operatorname{Re}\left(\rho^{\prime} \beta^{*}\right)$ and $\gamma \stackrel{\text { def }}{=}\left(1-\left|\rho^{\prime}\right|^{2}\right)\left(1-|\beta|^{2}\right)$ and the associated eigenvectors $\mathbf{v}_{k}=\left[\left\|\mathbf{a}_{k}\right\|^{2} \sigma_{s}^{2}\left(1+\rho^{*} \beta\right)-\lambda_{k}\right] \mathbf{a}_{1}-$ $\left\|\mathbf{a}_{k}\right\|^{2} \sigma_{s}^{2}\left(\rho^{\prime *}+\beta^{*}\right) \mathbf{a}_{2}$. Plugging these eigenvalues and eigenvectors in $\mathbf{U}=\left(\frac{\sigma_{n}^{2}}{\lambda_{1}}+\frac{\sigma_{n}^{4}}{\lambda_{1}^{2}}\right) \frac{\mathbf{v}_{1} \mathbf{v}_{1}^{H}}{\left\|\mathbf{v}_{1}\right\|^{2}}+\left(\frac{\sigma_{n}^{2}}{\lambda_{2}}+\frac{\sigma_{n}^{4}}{\lambda_{2}^{2}}\right) \frac{\mathbf{v}_{2} \mathbf{v}_{2}^{H}}{\left\|\mathbf{v}_{2}\right\|^{2}}$ and $\mathbf{U}^{\prime}=\frac{\sigma_{n}^{4}}{\lambda_{1}^{2}} \frac{\mathbf{v}_{1} \mathbf{v}_{1}^{H}}{\left\|\mathbf{v}_{1}\right\|^{2}}+\frac{\sigma_{n}^{4}}{\lambda_{2}^{2}} \frac{\mathbf{v}_{2} \mathbf{v}_{2}^{H}}{\left\|\mathbf{v}_{2}\right\|^{2}}$ and after cumbersome, but straightforward algebraic manipulations, expressions $\mathbf{a}_{k}^{H} \mathbf{U} \mathbf{a}_{k}$ and $\mathbf{a}_{k}^{H} \mathbf{U}^{\prime} \mathbf{a}_{k}$ w.r.t. the parameters $\sigma_{s}^{2}, \sigma_{n}^{2}, \rho$ and $\beta$ are deduced and Result 7 is proved.

\section{REFERENCES}

[1] H. Krim and M. Viberg, "Two decades of array signal processing research: The parametric approach," IEEE Signal Processing Mag., vol. 13, pp. 67-94, April 1996.

[2] A. B. Gershman, M. Rbsamen, and M. Pesavento, "One- and two-dimensional direction-of-arrival estimation: An overview of search-free techniques," Signal Processing, vol. 90, no. 5, pp. 1338-1349, 2010.

[3] H. Abeida and J.-P. Delmas, "Stochastic Cramér-Rao bound for noncircular signals with application to DOA estimation," IEEE Trans. Signal Process., vol. 52, no. 11, pp. 3192-3299, Nov. 2004.

[4] P. Gounon, C. Adnet, and J. Galy, "Localisation angulaire de signaux non circulaires," Traitement du Signal, vol. 15, no. 1 pp. 17-23, 1998.

[5] P. Chargé, Y. Wang and J. Saillard, “A non-circular sources direction finding method using polynomial rooting," Signal Processing, vol. 81, pp. 1765-1770, 2001.

[6] A. Zoubir, P. Chargé, and Y. Wang "Non-circular sources localization with ESPRIT," in Proc. ECWT, Munich, Germany, Oct. 2003.

[7] M. Haardt and F. Roemer, "Enhancements of unitary ESPRIT for non-circular sources," Proc. ICASSP, Montreal, Canada, May 2004.

[8] H. Abeida and J.-P. Delmas, "MUSIC-like estimation of direction of arrival for noncircular sources," IEEE Trans. Signal Process., vol. 54, no. 7, pp. 2678-2690, Jul. 2006.

[9] H. Abeida and J.-P. Delmas, "Statistical performance of MUSIC-like algorithms in resolving noncircular sources," IEEE Trans. Signal Process., vol. 56, no. 9, pp. 4317-4329, Sep. 2008. 
[10] J. Steinwandt, F. Roemer, and M. Haardt, ”Analytical ESPRIT-based performance study: What can we gain from non-circular sources?" in Proc. SAM, A Coruña, Spain, June 2014

[11] J. Steinwandt, F. Roemer, and M. Haardt, Performance analysis of ESPRIT-type algorithms for non-circular sources," in Proc. ICASSP, Vancouver, Canada, May 2013.

[12] F. Gao, A. Nallanathan, and Y. Wang, "Improved MUSIC under the co-existence of both circular and non-circular sources," IEEE Trans. Signal Process., vol. 56, no. 7, pp. 3033-3038, Jul. 2008.

[13] J. Steinwandt, F. Roemer, and M. Haardt, "ESPRIT-type algorithms for a received mixture of circular and strictly non-circular signals" Proc. ICASSP, Brisbane, Australia, April 2015.

[14] S. B. Hassen, F. Bellili, A. Samet, and S. Affes, "DOA estimation of temporally and spatially correlated narrowband noncircular sources in spatially correlated white noise," IEEE Trans. Signal Process., vol. 59, no. 9, pp. 4108-4121, Sept. 2011.

[15] Z. Liu, Z. Huang, Y. Zhou, and J. Liu, "Direction-of-arrival estimation of noncircular signals via sparse representation," IEEE Trans. Aerosp. Electron. Syst., vol. 48, no. 3, pp. 2690-2698, Jul. 2012.

[16] J. Xie, H. Tao, X. Rao, and J. Su, "Efficient method of passive localization for near-field noncircular sources," IEEE Ant. Wireless Propag. Lett., vol. 14, pp. 1223-1226, Feb. 2015.

[17] H. Chen, W. F. Wang, and W. Liu, "Joint DOA, range, and polarization estimation for rectilinear sources with a COLD array," IEEE Wireless Commun. Letters, vol. 8, no. 5, pp. 1398-1401, Oct. 2019.

[18] H. Chen, C. P. Hou, W. Liu, W. P. Zhu, and M. N. S. Swamy, "Efficient two-dimensional direction of arrival estimation for a mixture of circular and noncircular sources," IEEE Sensors Journal, vol. 16, no. 8, pp. 2527-2536, April 2016.

[19] H. Chen, C. P. Hou, W. P. Zhu, W. Liu, Y. Y. Dong, and Q. Wang, "ESPRIT-like two-dimensional direction finding for mixed circular and strictly noncircular sources based on joint diagonalization," Signal Processing, vol.141, pp. 48-56, Dec. 2017.

[20] E. Ollila, D. Tyler, V. Koivunen, and H. Poor, "Complex elliptically symmetric distributions: Survey, new results and applications," IEEE Trans. Signal Process., vol. 60, no. 11, pp. 5597-5625, Nov. 2012.

[21] P. Tsakalides and C. Nikias, "The robust covariation based MUSIC (roc MUSIC) algorithm for bearing estimation in impulsive noise environments," IEEE Trans. Signal Process., vol. 44, no. 7, pp. 1623-1633, July 1996.

[22] E. Ollila and V. Koivunen, "Robust antenna array processing using $M$-estimators of pseudo covariance," 14th lntermational Symposium on Personal Indoor and Mobile Radio Communication, 2003.

[23] M. Mahot, F. Pascal, P. Forster, and J.P. Ovarlez, "Asymptotic properties of robust complex covariance matrix estimates," IEEE Trans. Signal Process., vol. 61, no. 13, pp. 3348-3356, July 2013.

[24] S. Visuri, H. Oja, and V. Koivunen, "Subspace-based direction of arrival estimation using nonparametric statistics," IEEE Trans. Signal Process., vol. 49, no. 9, pp. 2060-2073, Sept. 2001.

[25] H. Abeida and J.-P. Delmas, "Slepian-Bangs formula and Cramer Rao bound for circular and non-circular complex elliptical symmetric distributions," IEEE Signal Process. Letters, vol. 26, no. 10, pp. 1561-1565, Oct. 2019.

[26] H. Abeida and J.-P. Delmas, "Robustness of subspace-based algorithms with respect to the distribution of the noise: Application to DOA estimation," Signal Processing, vol. 164, pp. 313-319, June 2019.

[27] C. El Kassis, J. Picheral, and C. Mokbel, "Advantages of nonuniform arrays using root-MUSIC," Signal Processing, vol. 90, no. 2, pp. 689-695, Feb. 2010.

[28] P. P. Vaidyanathan and P. Pal, "Direct-MUSIC on sparse arrays," in Proc. IEEE International Conference on Signal Processing and Communications (SPCOM), Bangalore, India, July 2012.

[29] H. Abeida and J.-P. Delmas, "Efficiency of subspace-based estimators for elliptical symmetric distributions," Signal Processing, vol. 174, October 2020. 
[30] J. T. Kent and D. E. Tyler, "Redescending $M$-estimates of multivariate location and scatter," Ann. Statist., vol. 19, no. 4, pp. 2102-2119, 1991.

[31] R. Maronna, "Robust $M$-estimators of multivariate location and scatter," Annals of Statistics, vol. 4, no. 1, pp. 51-67, Jan. 1976.

[32] D. E. Tyler, "A distribution-free estimator of multivariate scatter," The Annals of Statistics vol. 615 no. 21 pp. $234-251,1987$.

[33] F. Pascal, P. Forster, J.-P. Ovarlez, and P. Larzabal, "Performance analysis of covariance matrix estimates in impulsive noise," IEEE Trans. Signal Process., vol. 56, no. 6, 2018, pp. 2206-2217, June 2008.

[34] P. Stoica and A. Nehorai, "MUSIC, maximum likelihood, and Cramer-Rao bound," IEEE Trans. Acoust. Speech, Signal Processing, vol. 37, no. 5, pp. 720-741, May 1989.

[35] P. Stoica and A. Nehorai, "Performance study of conditional and unconditional direction of arrival estimation," IEEE Trans. Acoust. Speech, Signal Processing, vol. 38, no. 10, pp. 1783-1795, Oct. 1990.

[36] P. Stoica and A. Nehorai, "MUSIC, maximum likelihood, and Cramer-Rao bound: Further results and comparisons," IEEE Trans. Acoust. Speech, Signal Processing, vol. 38, no. 12, pp. 2140-2150, Dec. 1990.

[37] A. J. Weiss and B. Friedlander, "On the Cramer-Rao bound for direction finding of correlated signals," IEEE Trans. Signal Process., vol. 41, no. 1, pp. 495-499, Jan. 1993.

[38] J.-P. Delmas, M.N. El Korso, H. Gazzah, and M. Castella, "CRB analysis of planar antenna array for optimizing near-field source localization," Signal Processing, vol. 127, pp. 117-134, March 2016.

[39] O. Besson and Y. Abramovich, "On the Fisher information matrix of multivariate elliptically contoured distributions," IEEE Signal Process. Letters, vol. 20, pp. 1130-1133, Nov. 2013.

[40] M. Greco and F. Gini, "Cramér-Rao lower bounds on covariance matrix estimation for complex elliptically symmetric distributions," IEEE Trans. Signal Process., vol. 61, no. 24, pp. 6401-6409, Dec. 2013.

[41] R.J. Serfling, Approximation Theorems of Mathematical Statistics, John Wiley and Sons, 1980. 